\title{
An in vitro bioengineered model of the human arterial neurovascular unit to study neurodegenerative diseases
}

\author{
Jerome Robert ${ }^{1,2,3^{*}}$ (D) Nicholas L. Weilinger ${ }^{2}$, Li-Ping Cao ${ }^{1,2,4}$, Stefano Cataldi ${ }^{4}$, Emily B. Button ${ }^{1,2}$, Sophie Stukas ${ }^{1,2}$, \\ Emma M. Martin ${ }^{1,2}$, Philip Seibler ${ }^{5}$, Megan Gilmour ${ }^{1,2}$, Tara M. Caffrey ${ }^{1,2}$, Elyn M. Rowe ${ }^{1,2}$, Jianjia Fan ${ }^{1,2}$, \\ Brian MacVicar ${ }^{2}$, Matthew J. Farrer ${ }^{4,6}$ and Cheryl L. Wellington ${ }^{1,2,7,8^{*}}$
}

\begin{abstract}
Introduction: The neurovascular unit (NVU) - the interaction between the neurons and the cerebrovasculature - is increasingly important to interrogate through human-based experimental models. Although advanced models of cerebral capillaries have been developed in the last decade, there is currently no in vitro 3-dimensional (3D) perfusible model of the human cortical arterial NVU.

Method: We used a tissue-engineering technique to develop a scaffold-directed, perfusible, 3D human NVU that is cultured in native-like flow conditions that mimics the anatomy and physiology of cortical penetrating arteries.

Results: This system, composed of primary human vascular cells (endothelial cells, smooth muscle cells and astrocytes) and induced pluripotent stem cell (iPSC) derived neurons, demonstrates a physiological multilayer organization of the involved cell types. It reproduces key characteristics of cortical neurons and astrocytes and enables formation of a selective and functional endothelial barrier. We provide proof-of-principle data showing that this in vitro human arterial NVU may be suitable to study neurovascular components of neurodegenerative diseases such as Alzheimer's disease (AD), as endogenously produced phosphorylated tau and beta-amyloid accumulate in the model over time. Finally, neuronal and glial fluid biomarkers relevant to neurodegenerative diseases are measurable in our arterial NVU model.

Conclusion: This model is a suitable research tool to investigate arterial NVU functions in healthy and disease states. Further, the design of the platform allows culture under native-like flow conditions for extended periods of time and yields sufficient tissue and media for downstream immunohistochemistry and biochemistry analyses.
\end{abstract}

\section{Background}

The brain consumes $\sim 20 \%$ of total body oxygen and glucose utilization despite representing only $2 \%$ of total body mass $[1,2]$. These high metabolic demands vary both temporally and spatially in the brain, and are met by the coordinated action of several cell types known collectively as the neurovascular unit (NVU) [3, 4]. Neural activity

\footnotetext{
* Correspondence: jerome.robert@usz.ch; cheryl.wellington@ubc.ca 'Department of Pathology and Laboratory Medicine, University of British Columbia, Vancouver, British Columbia V6T 1Z3, Canada

Full list of author information is available at the end of the article
}

increases local cerebral blood flow (CBF) through a process known as neurovascular coupling [5]. This process links neuronal glutamate release to neuronal nitric oxide (NO) secretion that modulates vascular tone of nearby smooth-muscle cells (SMC) in the arterioles, as well as ATP-triggered astrocyte calcium waves that regulate the release of vasoactive molecules that modulate vascular tone of adjacent pericytes in the capillaries [6-9]. Endothelial cells (EC) within the NVU form the blood-brain barrier (BBB) that restricts blood-brain exchange and regulates brain waste excretion [10]. Disease-associated 
changes in $\mathrm{CBF}$ and the $\mathrm{BBB}$ are observed in many neurodegenerative disorders including Alzheimer's disease (AD) $[3,11]$. As such, there is tremendous interest in developing cell-based models that mimic the BBB and NVU. Such models would greatly facilitate gaining a better understanding of the interactions between neurons and the vasculature in both physiological and pathophysiological conditions. If made with human cells, they would also provide an invaluable translational platform for the development of neurotherapeutics.

Tissue engineering, organoid culture, and microfluidic technologies have emerged in the last decades as powerful research tools to study how different cell types interact in the context of their native extracellular matrices (ECM), thus driving next-generation models of human disease [12-14]. Among the many models relevant to the central nervous system developed thus far, the most advanced include: i) The Parker model that links a BBB microfluidic chip containing EC, pericytes and astrocytes to a brain microfluidic chip composed of neurons and astrocytes via artificial cerebrospinal fluid perfusion [13]. ii) The Svendsen model consisting of a single microfluidic chip where the vascular chamber of EC is separated from the brain chamber consisting of neurons, astrocytes and pericytes by a semi-permeable membrane [15]. These groups, and many others, have focused their efforts on modeling the microvasculature, given the importance of brain capillaries in neuronal function and the need for better models to assess drug uptake across the $\mathrm{BBB}$.

By contrast, we aimed to develop a model of the large arterial NVU, as cerebral arteries and arterioles regulate many physiological and pathophysiological processes important for brain function $[16,17]$. We recently developed a human cerebrovascular model consisting of primary EC and SMC cultured with or without astrocytes, mimicking penetrating and leptomeningeal arteries, respectively [12]. Using this model we demonstrated the possibility to study key vascular pathophysiological features of $\mathrm{AD}$ in vitro, namely the accumulation of beta amyloid $(A \beta)$ in the vascular wall and subsequent vascular inflammation, which comprises cerebral amyloid angiopathy (CAA) $[12,18,19]$. However, the previously reported model lacked neurons and relied solely on exogenous recombinant $A \beta$, and thus, is limited in its ability to study the role of neuronal biology and neurovascular function.

In the present study, we describe expansion of this platform to generate a model of the arterial NVU composed of primary human EC, SMC and astrocytes cultured in the presence of human induced pluripotent stem cells (iPSC)-derived glutamatergic cortical neurons. Under luminal native-like flow conditions, this method creates perfusible vessels that can be sampled from both the "brain" and "blood" sides. Histological analyses confirmed a multi-layer structure similar to native human cerebrovascular tissues, and biochemical analysis confirmed the presence of a tight endothelial barrier separating a closed "brain" compartment from a separate "blood" compartment that circulates through the vessel lumen. We further showed that iPSC-derived neurons cultured in this bioengineered arterial NVU were electrically excitable and could both secrete glutamate and had measurable $\alpha$-amino-3-hydroxy-5-methyl-4-isoxazolepropionic acid receptor (AMPAR) currents, suggesting possible synapse formation. Finally, we confirmed the potential to study key elements of arterial pathophysiology relevant to $A D$ in vitro, as endogenous $A \beta$ peptides were produced by neurons and transported from the "brain" compartment to the "blood" compartment, where they gradually accumulated in the vascular wall with greater deposition of $A \beta 40$ than $A \beta 42$. Endogenous phosphorylated tau was also confirmed to deposit in the vascular wall. The model described here thus serves as a controlled platform that can be used to interrogate the physiology of the human arterial NVU, including the possibility of measuring tau, neurofilament light (NF-L), glial fibrillary acidic protein (GFAP), and ubiquitin carboxyl-terminal hydrolase L1 (UCH-L1) as brain biomarkers.

\section{Methods}

\section{Culture of iPSC-derived neurons}

Glutamatergic cortical neurons were derived from human iPSC using a modified protocol from Shi et al [20] Briefly, iPSC (line L2131 [21]) were maintained in mTesRTM1 medium (StemCell). Seven days after the last passage, iPSC were groomed by removing any colonies having an appearance of differentiated cells, irregular borders or a transparent-center. IPSC were washed with Dulbecco's Modified Eagle Media (DMEM)/F12 (Invitrogen), dissociated into single cells using accutase (Invitrogen) and filtered through a $0.45 \mu \mathrm{m}$ cell strainer. After 2 washes with IPS media (4:5 DMEM/F12, 1:5 knockout serum replacement [KOSR], $15 \mathrm{mM}$ HEPES, $1 \%$ glutamine, 1\% MEMnon essential amino acids [NEAA], $0.1 \mathrm{mM} \quad \beta$ mercaptoethanol, $10 \mathrm{ng} / \mathrm{mL}$ human fibroblast growth factor 2 [hFGF2]), iPSC were plated at a density $<200,000$ cells $/ \mathrm{cm}^{2}$ on gelatin-coated plates in IPS medium containing $10 \mu \mathrm{M}$ ROCK-Inhibitor (Y-27632, Stemcell Technologies). After $1 \mathrm{~h}$ at $37^{\circ} \mathrm{C}$, non-adherent cells were collected and suspended in murine embryonic fibroblast (MEF) conditioned media containing $10 \mu \mathrm{M} \mathrm{Y}-27632$ and $20 \mathrm{ng} /$ $\mathrm{mL}$ of human FGF and plated on Matrige ${ }^{\circ}$ matrix (Corning)-coated plates at a density of $1-1.5 \times 10^{6}$ per 6 -well plate. MEF medium was changed daily until cells were 95\% confluent, which was usually after 1 day. To initiate neuronal differentiation, $2 \mathrm{~mL}$ of KSR (Knockout DMEM 
with $15 \%$ KOSR, $1 \%$ glutamine, $1 \%$ MEM-NEAA, $0.1 \mathrm{mM}$ $\beta$-mercaptoethanol) were added to the cells for 4 days. On day 5 , KSR media was gradually replaced by neural maintenance medium (NMM: 1:2 DMEM/F12, 1:2 neurobasal medium, $0.25 \% \mathrm{~N} 2$ supplement, $0.25 \mu \mathrm{g} / \mathrm{mL}$ insulin, $0.5 \%$ MEM-NEAA, $50 \mu \mathrm{M}$ M2-ME, $1 \%$ neuroCult SM1 Neuronal supplement, $1 \%$ glutamine, $1 \%$ Pen/strep) medium at a ratio of 3:1 KSR:NMM on day $5,2: 2$ on day 7, 1:3 on day 9, and $100 \% \mathrm{NMM}$ containing $1 \mu \mathrm{M}$ dorsomorphine, $10 \mu \mathrm{M}$ transforming growth factor- $\beta$ (TGF- $\beta$ ) inhibitor SB $431542,10 \mu \mathrm{M}$ Y-27632 on day 11 . On day 12 of differentiation, medium was removed and tissue was dissociated into clumps using a pipette. Cells were plated onto $6 \mathrm{~cm}$ dishes coated with poly-D-lysine/laminin in NMM. On day 13-17 of differentiation, media was removed and NMM supplemented with $20 \mathrm{ng} / \mathrm{mL}$ human FGF and 20 $\mathrm{ng} / \mathrm{mL}$ of human brain derived neurotrophic factor (BDNF) was added. On day 18 of differentiation, rosettes were manually picked with a sterile pipette and plated in NMM supplemented with $20 \mathrm{ng} / \mathrm{mL}$ of BDNF, $20 \mathrm{ng} / \mathrm{mL}$ of glial derived neurotrophic factor (GDNF), and $0.2 \mathrm{mM}$ ascorbic acid on poly-D-lysine/laminin-coated 6-well plates. From day 19-22 of differentiation, media was fully refreshed every other day. On day 23 of differentiation, rosettes were again manually picked with a sterile pipette and plated in NMM supplemented with $20 \mathrm{ng} / \mathrm{mL}$ of BDNF, $20 \mathrm{ng} / \mathrm{mL}$ of GDNF, and $0.2 \mathrm{mM}$ ascorbic acid on poly-D-lysine/laminin-coated 6-well dishes. From day 2427 of differentiation, medium was refreshed every other day. On day 28 of differentiation, medium was removed, cells were washed with PBS and dissociated using accutase. After 10-15 min, cells were lifted by pipetting up and down before collecting and centrifuging at $160 \mathrm{~g}$ for 5 min. Cells were suspended in complete NMM and plated on poly-D-lysine/laminin coated 6-well dishes at a density of $1 \times 10^{6}$ cells per well. From day 29 of differentiation on, medium was refreshed with full NMM every 3 days. IPSCderived neurons were genotyped as described [22] as apoEs3/4 .

\section{Isolation and culture of vascular cells}

All experiments were conducted under an approved clinical protocol (UBC Clinical Ethics Research Board H13-02719) after obtaining written informed consent. Human umbilical vein endothelial cells (EC) and human umbilical cord myofibroblasts (SMC) were isolated as described [23]. Briefly, EC were isolated using the instillation method, where the vein lumen was filled with a solution of collagenase $(2 \mathrm{mg} / \mathrm{mL}$, Collagenase A, Roche) in serum-free DMEM (Invitrogen) before clamping both ends. After $20 \mathrm{~min}$ at $37^{\circ} \mathrm{C}$, Advanced DMEM (Gibco) supplemented with $1 \%$ L-glutamine, $0.05 \%$ penicillin/ streptavidin (pen/strep) and 10\% fetal bovine serum (FBS) (Invitrogen) was flushed through the lumen and the cell suspension was centrifuged at $1200 \mathrm{rpm}$ for 5 min. EC were expanded in full endothelial growth medium (EGM ${ }^{\mathrm{sm}}-2$, LONZA Inc.), supplemented with vascular endothelial growth factor (VEGF), human recombinant insulin-like growth factor-1 (hrIGF-1), human epidermal growth factor (hEGF), amphotericin-B, hydrocortisone, ascorbic acid, heparin, and 2\% (FBS) up to passage 10 with media changed every 3-4 days. SMC were isolated by mincing the vessel wall into small pieces $(\sim 2-3 \mathrm{~mm})$ and incubating at room temperature for $20 \mathrm{~min}$ without medium under sterile laminar flow to ensure physical attachment of the pieces. Advanced DMEM (Invitrogen) supplemented with 1\% L-glutamine, $0.05 \%$ pen/strep and $10 \%$ FBS was subsequently added to the minced vessels and adherent cells were expanded up to passage 10 with media changed every 3-4 days. EC and SMC from different donors were genotyped as

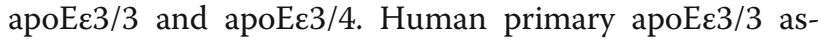
trocytes (Sciencell) were cultivated in astrocyte media (Sciencell) supplemented with astrocyte growth factor (Sciencell), 0.05\% pen/strep and 2\% FBS up to passage 5 with media changed every $3-4$ days.

\section{Bioengineering the in vitro arterial NVU}

Bioengineered constructs were fabricated using a dynamic, semi-pulsatile flow bioreactor system. Tubular biodegradable scaffolds (length $1.5 \mathrm{~cm}$ and inner diameter $2 \mathrm{~mm}$ ) were produced as previously described [12]. Briefly, non-woven polyglycolic acid (PGA, Biomedical Structure) meshes (thickness: $1 \mathrm{~mm}$ and density: $70 \mathrm{mg} /$ cc) were dip-coated with polycaprolactone (PCL, Sigma Aldrich) by dipping PGA mesh in a solution of $1.75 \%$ (w/w) PCL/tetrahydrofuran (THF) solution (Sigma Aldrich), shaping into tubes using heat, and externally coating with a $10 \%$ PCL/THF (w/w) solution. Scaffolds were sterilized by immersion in $70 \%$ ethanol for $30 \mathrm{~min}$, followed by three PBS washes and finally immersion in advanced DMEM supplemented with 10\% FBS for at least $12 \mathrm{~h}$. Confluent SMC were washed with PBS, lifted from a $10 \mathrm{~cm}$ plate using $1 \mathrm{~mL}$ of trypsin (GIBCO, 5 min. $37^{\circ} \mathrm{C}$ ) and collected using $3 \mathrm{~mL}$ Advanced DMEM followed by $5 \mathrm{~min}$ of centrifugation at $300 \mathrm{~g}$. The supernatant was removed and 2-3 $\times 10^{6} \mathrm{SMC}$ were suspended in $15 \mu \mathrm{L}$ of thrombin (Sigma Aldrich $100 \mathrm{mU} / \mathrm{mL}$ PBS). $15 \mu \mathrm{L}$ of fibrinogen (Sigma Aldrich 15 fibrinogen, $10 \mathrm{mg}$ clottable protein $/ \mathrm{mL}$ in $\mathrm{PBS}$ ) was then added to the thrombin/SMC and the mixed solution was seeded on the inner surface of the scaffold to a final density of 2$3 \times 10^{6}$ cells $/ \mathrm{cm}^{2}$. The seeded scaffold was incubated under static conditions in Advanced DMEM supplemented with $10 \% \mathrm{FBS}, 1 \% \mathrm{~L}$-glutamine and $0.05 \%$ pen/ strep and $1.5 \mathrm{mM} \mathrm{L-ascorbic} \mathrm{acid} \mathrm{(Sigma} \mathrm{Aldrich).} \mathrm{After}$ 3 to 5 days, advanced DMEM supplemented with $10 \%$ FBS, $1 \%$ L-glutamine and $0.05 \%$ pen/strep and ascorbic 
acid was flowed through the lumen of the vessel using a peristaltic pump to mimic blood flow for 7 days. Confluent EC were washed with $\mathrm{PBS}$, lifted from a $10 \mathrm{~cm}$ plate using $1 \mathrm{~mL}$ of trypsin $\left(5 \mathrm{~min}\right.$ at $\left.37^{\circ} \mathrm{C}\right)$ and collected in 3 $\mathrm{mL}$ of Advanced DMEM/ containing 10\% FBS per plate follow by $5 \mathrm{~min}$ of centrifugation at $300 \mathrm{~g}$. After removing the supernatant, EC were suspended in complete EGM2 containing $10 \% \mathrm{FBS}$ at a density of $40 \times 10^{6}$ cells/ $\mathrm{mL}$. Vascular intermediates were then seeded with EC to a final density of $1 \times 10^{6}$ cells $/ \mathrm{cm}^{2}$ on the luminal side and cultivated first in static conditions in full $E G M^{\mathrm{nm}}-2$ supplemented as above. After 3 days, confluent astrocytes were washed with $\mathrm{PBS}$, lifted from a $10 \mathrm{~cm}$ plate using $1 \mathrm{~mL}$ of trypsin $\left(5 \mathrm{~min}\right.$ at $\left.37^{\circ} \mathrm{C}\right)$ and collected in 3 $\mathrm{mL}$ of Advanced DMEM containing 10\% FBS per plate follow by centrifugation at $300 \mathrm{~g}$ for $5 \mathrm{~min}$. After removing the supernatant, $1 \times 10^{6}$ astrocytes were suspended in $10 \mu \mathrm{L}$ of thrombin (as above), then mixed with $10 \mu \mathrm{L}$ of fibrinogen (as above) and directly seeded on the antelumen side of the tissue at a density $1 \times 10^{6} \mathrm{cells} / \mathrm{cm}^{2}$. After $5 \mathrm{~min}$ at $\mathrm{RT}$, tissue constructs were placed in complete astrocyte media under static conditions. After $24 \mathrm{~h}$, confluent iPSC-derived neurons (age 60 to 80 days) were washed twice with PBS, lifted from a $10 \mathrm{~cm}$ plate using $1 \mathrm{~mL}$ of acutase (GIBCO, $10 \mathrm{~min}$ at $37^{\circ} \mathrm{C}$ ) and collected in $3 \mathrm{~mL}$ of completed NMM media per plate followed by centrifugation at $300 \mathrm{~g}$ for $5 \mathrm{~min}$. The supernatant was removed and neurons were suspended in $10 \mu \mathrm{L}$ ice cold Matrigel $^{\circ}$ matrix as a cell carrier. Neurons were seeded on the antelumen side of the engineered vessel at a density of $2 \times 10^{6}$ cells $/ \mathrm{cm}^{2}$. Tissues were maintained at RT for 5 min until gelation of the Matrigel $^{\triangleright}$ matrix was complete before mounting in the bioreactor with completed NMM media both in the tissue (with Y-27632) and circulation chamber. Tissues were maintained under flow conditions for a maximum of 21 days before experiments.

\section{Green fluorescent protein (GFP) electroporation}

Neurons were transfected with the pmaxGFP vector (Lonza) using the Nucleofector 2b (Lonza) device. Briefly, neurons were washed twice with PBS and detached by adding accutase to the wells for 5 to $15 \mathrm{~min}$. Neurons were collected in NMM+ as described above and centrifuged at $250 \mathrm{~g}$ for $3 \mathrm{~min}$. They were then suspended in Mouse NSC Nucleofector Solution (Lonza) at a density of $4 \times 10^{6}$ neurons $/ 100 \mu \mathrm{L}$ with $4 \mu \mathrm{g}$ of pmaxGFP, followed by transfection in the Nucleofector $2 \mathrm{~b}$ using the program B-016. $500 \mu \mathrm{L}$ warm $\mathrm{NMM}+$ was then directly added to the transfected cells. After $5 \mathrm{~min}$, cells were centrifuged at $250 \mathrm{x}$ g at room temperature for $3 \mathrm{~min}$, suspended in $10 \mu \mathrm{L}$ Matrigel $^{\circ}$ matrix and 10 $\mu \mathrm{L}$ NMM with Y-27632 and seeded on the antelumen of the tissues as above.

\section{Electrophysiology}

Bioengineered tissues were carefully cut longitudinally in thirds, and transferred to a recording chamber continually perfused (1-2 mL/min) with artificial cerebral spinal fluid (aCSF) consisting of: $126 \mathrm{nM} \mathrm{NaCl}, 26 \mathrm{mM} \mathrm{NaHCO}_{3}, 2.5$ $\mathrm{mM} \mathrm{KCl}, 1.25 \mathrm{mM} \mathrm{NaH}{ }_{2} \mathrm{PO}_{4}, 2 \mathrm{mM} \mathrm{MgCl}, 2 \mathrm{mM} \mathrm{CaCl}_{2}$, and $10 \mathrm{mM}$ glucose. aCSF was continuously bubbled with $95 \% \mathrm{O}_{2} / 5 \% \mathrm{CO}_{2}$ and warmed to $33^{\circ} \mathrm{C}$ using a stage heater (Luigs \& Neumann). Whole-cell patch clamp recordings were obtained using thin-walled borosilicate glass microelectrodes (Warner) pulled to a tip resistance of 3-5 M 2 with a P-97 Flaming/Brown Micropipette Puller (Sutter Instruments). Electrodes were filled with an intracellular recording solution containing: $108 \mathrm{mM} \mathrm{K}$-gluconate, 3 $\mathrm{mM} \mathrm{KCl}, 2 \mathrm{mM} \mathrm{MgCl}$, $8 \mathrm{mM}$ Na-gluconate, $1 \mathrm{mM} \mathrm{K}_{2}$ EGTA, $230 \mu \mathrm{M} \mathrm{CaCl}_{2}, 50 \mu \mathrm{M}$ Alexa-594, $4 \mathrm{mM} \mathrm{K}_{2}$-ATP, $200 \mu \mathrm{M}$ Rhod-2 tripotassium salt (ThermoFisher), and $300 \mu \mathrm{M} \mathrm{Na}_{3}$-GTP at pH 7.25 with $10 \mathrm{mM}$ HEPES. Recordings were made using a MultiClamp 700B amplifier and a Digidata 1440A digitizer (Axon Instruments, Molecular Devices) controlled via Clampex 10.7 acquisition software. Cells were voltage clamped at $-60 \mathrm{mV}$ for glutamate puff experiments and passively current clamped (i.e. passive membrane potential monitoring) for $\mathrm{Ca}^{2+}$-imaging experiments. Stimulation trains $(200 \mathrm{pA}, 5 \mathrm{~ms} /$ pulse, $20 \mathrm{~Hz}, 5 \mathrm{~s}$ total) were applied for transient membrane depolarizations to trigger $\mathrm{Ca}^{2+}$ entry. Access resistance was always < $20 \mathrm{M} \Omega$. Glutamate $(200 \mu \mathrm{M})$ was transiently applied (5 s puffs) by using a puff electrode connected to a Picospritzer II (General Valve Corporation). The relative magnitude of AMPAR currents were quantified as normalized charge (i.e. the area under the curve) to control for the variability of the peak current responses. 6-cyano-7-nitroquinoxaline-2,3-dione (CNQX; $10 \mu \mathrm{M})$ was bath applied and was purchased from Tocris.

\section{Two-photon microscopy}

All experiments were performed on a LSM MP710 2photon imaging system (Zeiss). Cells were identified for whole-cell patch clamp and imaging using either widefield infrared illumination captured with a DAGE IR1000 camera (DAGE-MTI). This was preferable to patching GFP-labelled cells due to the sparse labeling and ease in identifying healthy neurons with transmitted illumination. GFP and/or Rhod-2 imaging was achieved by 2-photon excitation with a Ti:Sapphire Chameleon Ultra II 2-photon laser (Coherent) tuned to $850 \mathrm{~nm}$. Images were acquired with a Zeiss $20 \mathrm{X}-\mathrm{W} / 1.0 \mathrm{NA}$ objective at a pixel resolution of either $512 \times 512$ or $256 \times 256$ for fast Rhod-2 $\mathrm{Ca}^{2+}$-imaging. Emission light was split with a $575 \mathrm{~nm}$ longpass filter, and green and red emissions were filtered with $535 / 50 \mathrm{~nm}$ and $630 / 75 \mathrm{~nm}$ bandpass filters, respectively (all from Chroma Tech). Emission light was collected with LSM BiG GaAsP detectors from 
Zeiss, and data were acquired using Zen software (Zeiss) and analyzed in Fiji.

\section{Glutamate quantification}

Media was removed and cultures received a treatment of $56 \mathrm{mM} \mathrm{KCl}$ or regular Hanks Buffered Salt Solution (HBSS) for $30 \mathrm{~min}$. Glutamate was measured by highpressure liquid chromatography (HPLC) coupled to electrochemical detection (ALEXYS Neurotransmitter platform, Antec). $5 \mu \mathrm{L}$ of sample was automatically injected (AS 110 Autosampler, Antec) onto an Acquity UPLC HSS T3 analytical column (1 mm inner diameter, $50 \mathrm{~mm}$ length; Waters) perfused at a flow rate of $200 \mu \mathrm{L} / \mathrm{min}$ (LC $110 \mathrm{~S}$ pump, Antec) with a mobile phase containing $50 \mathrm{mM}$ phosphoric acid, $50 \mathrm{mM}$ citric acid, $0.1 \mathrm{mM}$ EDTA, and 2\% acetonitrile (pH 3.5). At the end of each sample, a solution of $50 \mathrm{mM}$ phosphoric acid, $50 \mathrm{mM}$ citric acid, $0.1 \mathrm{mM}$ EDTA, and 50\% acetonitrile (pH 3.5) was run to flush the column before the next sample. Each sample was mixed with a solution of $0.025 \mathrm{~g}$ of ortho-phthalaldehyde (a derivatization agent) in $250 \mu \mathrm{L}$ of methanol, $250 \mu \mathrm{L}$ of $1 \mathrm{M}$ sodium sulfite, and $4.5 \mathrm{~mL}$ of $0.1 \mathrm{M}$ borate buffer ( $\mathrm{pH} 10.4)$, for analytic detection. Glutamate was detected by means of an electrochemical detector (Decade II, Antec) with the cell potential set at $0.85 \mathrm{~V}$ vs. salt bridge. Retention times were $3.1 \pm 0.4 \mathrm{~min}$.

\section{ApoE measurement}

Secreted apoE levels in both the tissue chamber and circulation media were quantified by an apoE ELISA protocol as described previously [24]. $1 \mu \mathrm{M}$ of the liver-Xreceptor agonist GW3965 or dimethyl sulfoxide (DMSO) vehicle control were circulated through the lumen for $96 \mathrm{~h}$ before collecting media. Fluorescence was read at $325_{\mathrm{Ex}} / 420_{\mathrm{Em}}$ on an Infinite ${ }^{\circ}$ Tecan M200 Pro plate reader (Tecan Life Science).

\section{Histology and immunohistochemistry}

Bioengineered arterial NVU were prepared for cryopreservation by being washed in PBS, cut in $5 \mathrm{~mm}$ crosssectional pieces and fixed in 4\% paraformaldehyde (PFA, Sigma Aldrich). After $30 \mathrm{~min}$, tissues were washed three times in PBS, cryopreserved in $20 \%$ sucrose PBS solution for a minimum of $60 \mathrm{~min}$, cut in half longitudinally, embedded in $5 \%$ bovine skin gelatin (Sigma Aldrich) and $20 \%$ sucrose in PBS and stored at $-80^{\circ} \mathrm{C}$ until further processed. Samples were processed on a cryotome (chamber $-30^{\circ} \mathrm{C}$ and object $-25^{\circ} \mathrm{C}$ ) to generate $20 \mu \mathrm{m}$ sections that represented a longitudinal cross-section of the arterial NVU. Samples were stored at $-80^{\circ} \mathrm{C}$ until analysis. De-identified human AD brain tissues (cortex Brodmann area 9) were received from the Harvard Brain Tissue Resource Center under UBC Clinical Research Ethics Board protocol C04-0595 and cut into $20 \mu \mathrm{m}$ sections using a cryotome. On the day of immunohistochemistry, sections were rehydrated in PBS for $2 \times 10$ min before blocking for $30 \mathrm{~min}$ in $5 \%$ goat serum and $1 \%$ BSA in PBS. For immunohistochemistry on fresh tissues, bioengineered arterial NVU were washed with PBS and fixed in 4\% PFA. After fixation, tissues were washed three times with PBS, and were cut in half longitudinally, mounted with the abluminal side facing upward (lumen facing the microscopy slide) and directly processed for staining. After staining (see below), tissues were covered with a coverslip to specifically image the antelumen.

For immunofluorescence, cryopreserved sections (arterial NVU and human brain sections) and fresh arterial NVU were blocked in 5\% donkey serum and $1 \%$ BSA in PBS for $30 \mathrm{~min}$ at RT, incubated overnight at $4{ }^{\circ} \mathrm{C}$ with specific antibodies against the endothelial markers platelet endothelial cell adhesion molecule (CD31, RRID: AB_31432, WM59 Biolegend, 1:50) and von Willebrand factor (vWF, RRID:AB_259543, SigmaAldrich, 1:200), the SMC marker $\alpha$-SM-actin ( $\alpha$ SMA RRID:AB_476856, 1A4 SigmaAldrich, 1:200), the astrocyte markers glial fibrillary acidic protein (GFAP, RRID: AB_880203, Abcam, 1:200), and aquaporin 4 (AQ4, RRDI:AB_ 2039734, Alomone Labs, 1:100), the neuronal markers microtubule-associated protein 2 (MAP 2, RRID:AB 776174, Abcam, 1:200), $\beta$-tubulin III ( $\beta$-tub III, RRID: AB_2256751, Tuj1, 1:200), synapsin I (Syn, RRID:AB 2200097, Abcam, 1:200), A $\beta$ markers $A \beta$ 1-16 (6E10, RRID: AB_2565328, ThermoFisher Scientific, 1:50) and A $\beta$ fibrils (OC fibril, RRID: AB_1977024, EMD Millipore, AB2286, 1:200) and phospho-Tau (AT8, RRID:AB 223648, ThermoFisher, 1:250). After three additional PBS washes, sections and fresh arterial NVU were incubated for $45 \mathrm{~min}$ at RT with anti-rabbit or anti-mouse Alexa-488 or Alex-594 secondary antibodies (Invitrogen). Finally, sections were washed three times in PBS and mounted in Prolong Diamond antifade containing DAPI (Invitrogen). Sections were imaged with an Axioscan inverted microscope (Zeiss) and fresh arterial NVU were imaged with an Axioscan inverted confocal microscope (Zeiss). Cryopreserved sections were stained for Haematoxylin and Eosin (Sigma Aldrich) following manufacture's instructions.

\section{$A \beta$ quantification}

Luminal media was collected from the circulation loop and abluminal media was collected from the tissue chamber. For tissue biochemistry, $5 \mathrm{~mm}$ cross-sectional rings of tissue were crushed using a manual pestle and lysed in $150 \mu \mathrm{L}$ radioimmunoprecipitation assay (RIPA) buffer (10 $\mathrm{mM}$ Tris pH 7.4, $150 \mathrm{mM} \mathrm{NaCl}, 1.0 \% \mathrm{NP}-40,1.0 \%$ sodium deoxycholate, $0.1 \%$ SDS and cOmplete protease inhibitor with EDTA (Roche)). After homogenization, tissue samples were centrifuged for $15 \mathrm{~min}$ at $14000 \mathrm{~g}$ at $4{ }^{\circ} \mathrm{C}$ and 
the RIPA soluble fraction was transferred to a new tube. $250 \mu \mathrm{L}$ of $5 \mathrm{mM}$ of guanidine (GuHCl, Sigma Aldrich) was added to the tissue pellet and incubated overnight at RT under constant agitation before centrifugation at $14000 \mathrm{~g}$ at $4{ }^{\circ} \mathrm{C}$ for $15 \mathrm{~min}$. RIPA soluble and $\mathrm{GuHCl}$ soluble fractions were stored at $-20^{\circ} \mathrm{C}$ until quantification. RIPA (soluble), $\mathrm{GuHCl}$ (insoluble) and media fractions were quantified without dilution using A $\beta 40$ (KHB3442, Life Tech) and A 342 (KHB3482, Life Tech) commercial ELISA kits. $A \beta$ levels in RIPA and GuHCL fractions were normalized to total protein concentration as measured by bicinchoninic acid (BCA) assay (Fisher) and $\mathrm{A} \beta$ levels in abluminal media were normalized to tissue chamber media volumes.

\section{Single molecule array for biomarker quantification}

Total tau, GFAP, neurofilament light (NF-L) and ubiquitin carboxyl-terminal hydrolase L1 (UCH-L1) were quantified in media from the tissue chamber (abluminal) and from the circulation loop using the Neurology 4-plex A assay (Quanterix,) using the Simoa HD-1 analyzer (Quanterix) following the manufacturer's guidelines. Abluminal and circulating media were diluted off-board 2500 and 20 fold, respectively.

\section{SDS-PAGE and Immunoblotting}

Tissues composed of EC, SMC and astrocytes without or with neurons were lysed in RIPA buffer with Phosphostop (Roche). After 20 min on ice, tissues were crushed using a manual pestle before centrifuging for $10 \mathrm{~min}$ at $12000 \mathrm{~g}$ at $4{ }^{\circ} \mathrm{C}$. Total protein was quantified using BCA assay. Equal amounts of total protein $(25 \mu \mathrm{g})$ were separated by $10 \%$ acrylamide SDS-PAGE, followed by electrophoretic transfer to polyvinylidene fluoride (PVDF) membranes (Millipore). Membranes were blocked for 1 $\mathrm{h}$ using 1\% BSA in Tris-buffered saline (TBS, $20 \mathrm{mM}$ TrisBase and $150 \mathrm{mM} \mathrm{NaCl}, \mathrm{pH} 7.5)$ containing $0.5 \%$ Triton X (TBST). Phosphorylated tau AT8 (RRID:AB_ 223648, ThermoFisher, 1:1000), CP13 (RRID:AB 2314223, kindly gifted by Dr. Peter Davies at LitwinZucker Research Center for The Study of Alzheimer's Disease and Memory Disorders, 1:1000) and PHF1 (RRID:AB_2315150, Dr. Peter Davies, 1:1000) and total tau DA9 (RRID:AB_2716723, Dr. Peter Davies, 1:1000) were immunodetected by incubating overnight in blocking buffer at $4{ }^{\circ} \mathrm{C}$. Membranes were washed extensively with TBST and incubated with anti-mouse, (1:1000, Jackson ImmunoResearch) secondary antibody in blocking buffer. After $1 \mathrm{~h}$, membranes were washed extensively with TBST, developed using enhanced chemiluminescence (ECL, Amersham) and imaged using ChemiDoc MP imager (Biorad). Band densitometry was quantified using Fiji.

\section{Endothelium integrity}

Restriction of paracellular transport was determined by measuring $4 \mathrm{kDa}$ FITC-dextran extravasation from the lumen to the tissue chamber as described [12]. Briefly $250 \mu \mathrm{g} / \mathrm{ml}$ of $4 \mathrm{kDa}$ FITC-dextran (Sigma Aldrich) was circulated through the bioengineered tissue lumen. After $2 \mathrm{~h}$ tissue media was collected and fluorescence was measured at RT on Infinite ${ }^{-}$Tecan M200 Pro plate reader $\left(492_{\mathrm{Ex}} / 518_{\mathrm{Em}}\right)$. The permeability coefficient $\left(\mathrm{P}_{\mathrm{app}}\right.$, $\mathrm{cm} / \mathrm{s}$ ) was calculated using the following equation: $\mathrm{P}_{\mathrm{app}}=$ $(\mathrm{dQ} / \mathrm{dt}) *\left(1 / \mathrm{A}^{*} \mathrm{C}_{0} * 60\right)$ where $\mathrm{dQ} / \mathrm{dt}$ is the amount of FITC-dextran transported per minute ( $\mathrm{ng} / \mathrm{min}$ ), A is the surface area of the tissue in $\mathrm{cm}, \mathrm{C}_{0}$ is the initial concentration of FITC-dextran $(\mathrm{ng} / \mathrm{ml})$ and 60 is the conversion from minutes to seconds.

\section{Statistics}

Comparisons between groups were performed using unpaired or paired Student t-test, or one-way ANOVA with Dunnett's or Sidack's multicomparison test. Dependence analyses were assessed through Pearson correlation analysis. Values below the detection limit of the ELISAs were considered as 0 for statistical analysis and plotted as gray points. Data were obtained from at least three independently seeded bioengineered arterial NVU and graphically represented as scatter or before-after plots with mean \pm standard error of the mean (SEM). $P$ values of $<0.05$ were considered statistically significant. All statistical analyses were performed using GraphPad Prism-5 or SPSS software.

\section{Results}

Production and anatomical characterization of bioengineered arterial NVU

Human arterial NVU were fabricated by sequentially seeding, in order, primary human SMC, primary human EC and primary human astrocytes into a tubular porous scaffold consisting of polyglycolic acid (PGA), and polycaprolactone (PCL), measuring $15 \mathrm{~mm}$ long and $2 \mathrm{~mm}$ in diameter as described previously [12]. $24 \mathrm{~h}$ after seeding astrocytes, iPSC-derived neurons (aged 60 to 80 days) were seeded on the antelumen of the tissues using Matrigel $^{\circ}$ matrix as a cell carrier. A schematic of the finished bioengineered arterial NVU and bioreactor system is provided in Fig. 1a and histology of a post-mortem endogenous human arterial NVU is provided in Sup. Fig 1. Specifically, after 3 weeks in culture under native-like luminal flow conditions, cryosections from bioengineered arterial NVU were prepared along the longitudinal axis showing a cross section of the bioengineered vessel wall and processed for immunofluorescence staining against the EC marker CD31, the SMC marker $\alpha \mathrm{SMA}$, and the astrocyte marker GFAP. We observed a multilayer tissue organization resembling a cerebral artery; with a single 
a

\section{BIOREACTOR SYSTEM NVU MODEL}
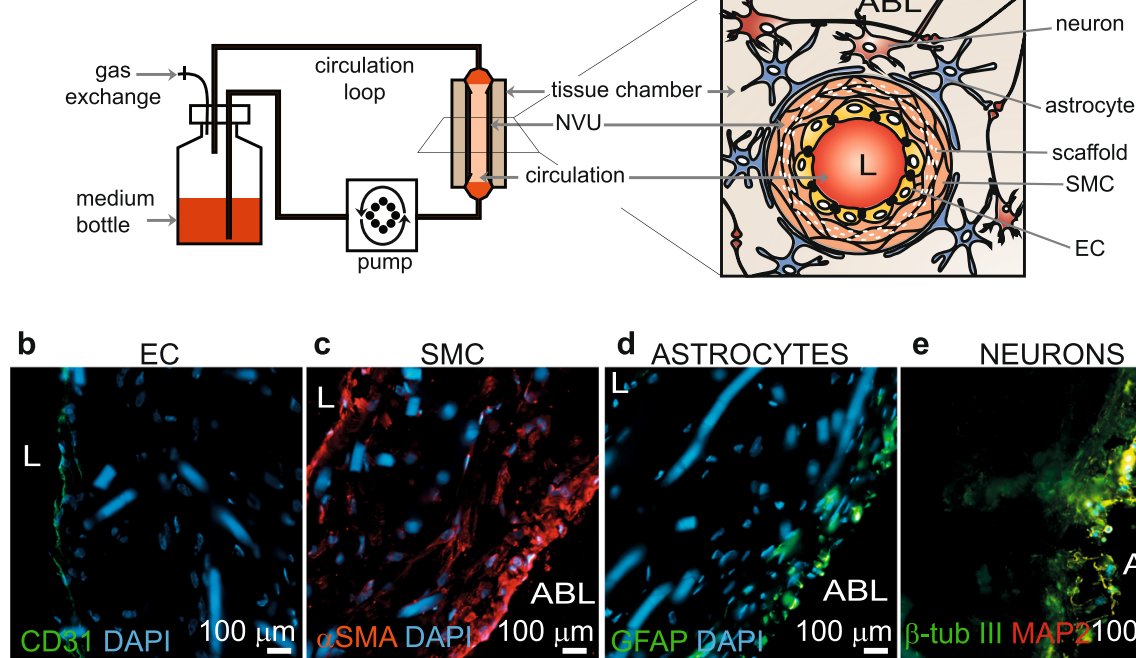

\section{d ASTROCYTES e NEURONS}

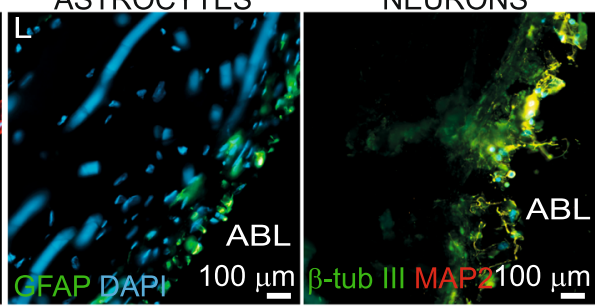

f NEURONS/ASTROCYTES
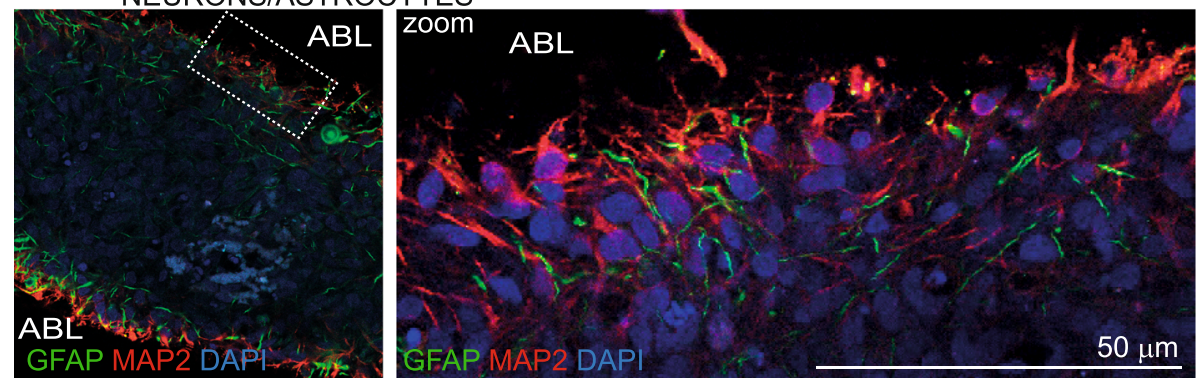

Fig. 1 Histological structure of bioengineered arterial NVU. a Schematic representation of the bioreactor and arterial NVU model. b-e Cryopreserved bioengineered arterial NVU were cut longitudinally to show a cross-section of the NVU wall. The expression of CD31 (b) confirmed the presence of an endothelial cell monolayer on the luminal side of the bioengineered NVU and ASMA (c) confirmed the smooth muscle phenotype of the cells in the inner layer. GFAP (d) and MAP 2/ß-tublll (e) positive staining confirmed respectively the astrocyte and neuron phenotype of the cells on the out layers in radial section of the NVU. f Bioengineered arterial NVU were stained without cryopreservation and were mounted directly on microscopy slide with the antelumen facing the coverslip. An optical sectioning was performed using confocal microscopy to image a focal plan . Staining against GFAP and MAP 2 confirmed the imbrication of the astrocytes and neurons. $L=$ lumen, $A B L=$ albumen

layer of EC on the lumen, multiple layers of SMC surrounding the endothelium and astrocyte layers on the antelumen (Fig. 1b-d). Immunofluorescence staining also demonstrated the presence of the neuronal marker $\beta$ tubulin-III and MAP 2 positive cells in the most abluminal layers of the bioengineered vessels, supporting the presence of neurons in the culture (Fig. 1e). The abluminal structure was further characterized by immunofluorescence staining against GFAP and MAP 2 by optical sectioning to image a focal plane in the abluminal side of a fresh bioengineered arterial NVU using confocal microscopy. Co-staining demonstrated that neurons and astrocytes form an imbricated network of cells on the last abluminal layers of cells with astrocytes penetrating farther into the tissue than neurons, whereas cells deeper in the tissue $(\sim 50 \mu \mathrm{m})$ were negative for both markers (Fig. 1f). Interestingly, contrary to cells grown in 2D culture, iPSC-derived neurons seeded on the bioengineered vessels do not form colonies but rather appear to be uniformly dispersed on the abluminal surface along astrocytes. Finally, H\&E staining of longitudinally cut cryosections showed formation of a multilayered tissue with remaining scaffold (arrows) in the core of the vascular wall (brownish features) and the formation of extremely dense layers on the albumen (Sup Fig. 2a).

\section{Cellular functionality in the bioengineered arterial NVU} Several approaches were used to evaluate neuronal function in the bioengineered arterial NUV. We first analysed synapsin-I expression using immunofluorescent staining of fresh arterial NVU. Confocal imaging confirmed punctate synapsin staining in MAP 2 positive cells (Fig. 2a, white arrows). Second, we measured glutamate release after $\mathrm{KCl}$ treatment using high performance liquid chromatography (HPLC) with electrochemical detection. After 
a

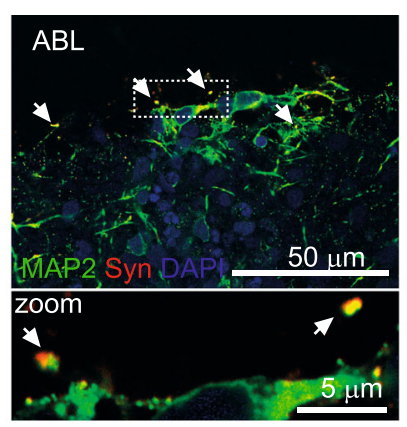

d

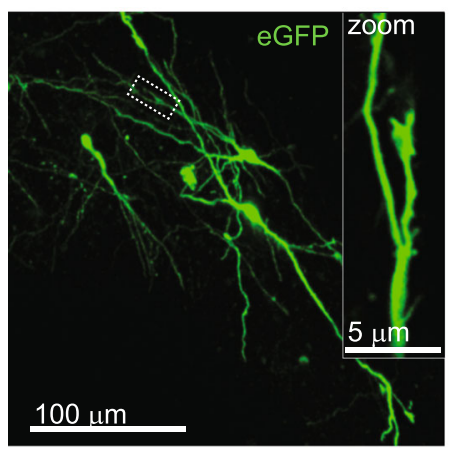

b

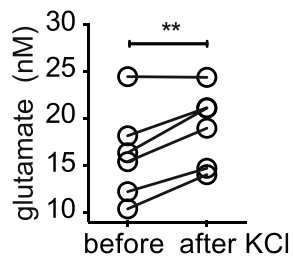

c

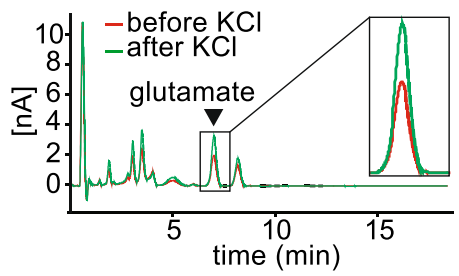

e
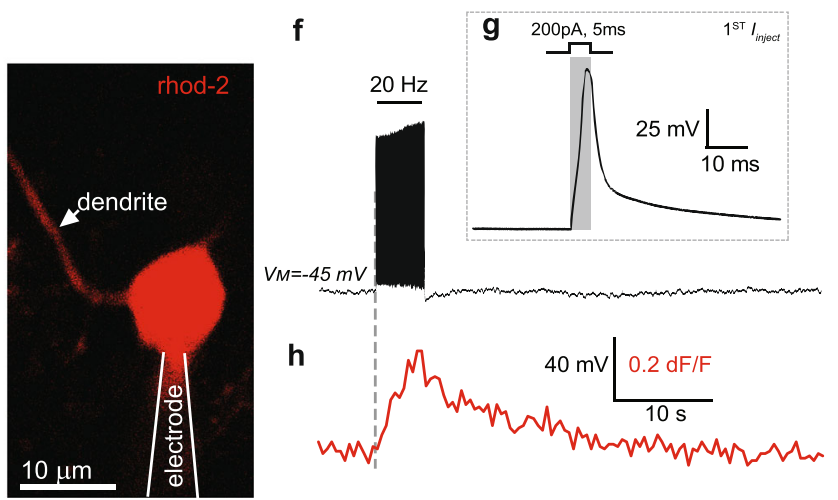

i

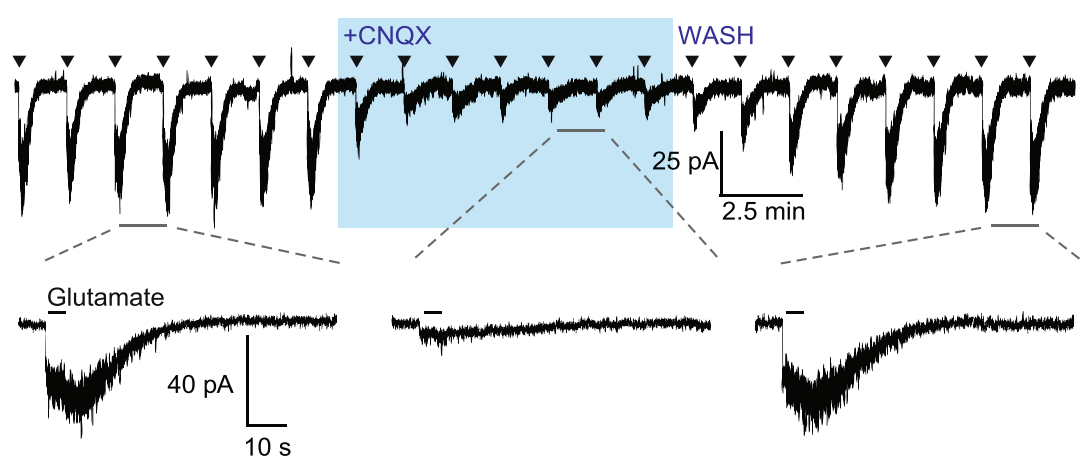

j

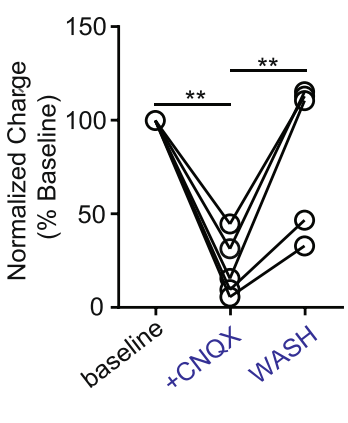

k

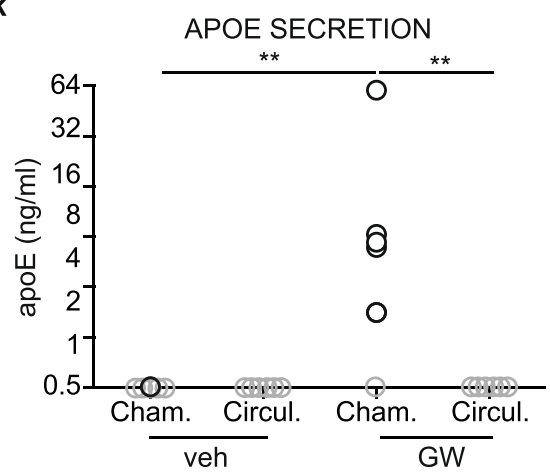

Fig. 2 (See legend on next page.) 
(See figure on previous page.)

Fig. 2 Depolarization- and glutamate-driven activity in abluminal cells indicates a neuronal phenotype and apoE secretion in the tissue chamber indicates astrocyte function and endothelial barrier formation. a Immunostaining against MAP 2 and synapsin-I (Syn) confirmed the presence of synapses in iPSC-derived neurons cultured in arterial NVU. b Glutamate release measured by HPLC showing increase after $\mathrm{KCl}$ treatment. $\mathbf{c}$ Example HPLC curves. d Two-photon Z-projection image of iPSC cells expressing eGFP. Dotted box displays region of zoomed inset, highlighting dendritic morphology and synaptic structure. e Example image of a whole-cell patch clamped iPSC-derived neuron dialyzed with the red $\mathrm{Ca}^{2+}{ }_{-}$ indicator Rhod-2. Proximal dendrites were imaged for depolarization-induced $\mathrm{Ca}^{2+}$-entry. $\mathbf{f}$ Representative current-clamp trace from patched cell in 'e' during $20 \mathrm{~Hz}$ spike train stimulation. g Single current injection (200 pA, $5 \mathrm{~ms}$ ) example from ' $\mathbf{f}$ ' showing change in membrane potential. $\mathbf{h}$ Time-correlated Rhod-2 signal from trace 'e' showing depolarization-induced $\mathrm{Ca}^{2+}$-increase. $\mathbf{i}$ (Top trace) Full-length voltage-clamp recording showing glutamate puff-evoked (triangles) AMPAR currents that were amenable to block by CNQX $(10 \mu \mathrm{M})$ and recovered in washout. (Bottom trace) Example AMPAR currents before, during, and after CNQX application. $\mathbf{j}$ Quantitative summary of normalized charge for glutamate inward currents in the presence of CNQX $\left(n=5,{ }^{*} P<0.01\right)$. $\mathbf{k}$ Astrocyte and endothelium barrier functions were confirmed by treating tissues with $1 \mu \mathrm{M}$ LXR agonist GW3965 for $96 \mathrm{~h}$ and measuring the levels of astrocyte-derived apoE secreted into the tissue chamber and circulation media. Values below the detection of the ELISA are plotted in gray. Points in graphed data represent individual bioengineered vessels, bars represent mean, error bars represent \pm SEM and analysed by one way ANOVA ** $P<0.01$

3 weeks under flow conditions, tissues were treated with $56 \mathrm{mM} \mathrm{KCl}$. After $30 \mathrm{~min}$, five of the six independent tissues with neurons tested demonstrated significant increased glutamate release (Fig. 2b-c). Next, we used twophoton microscopy and electrophysiology to assess the morphology and electrical properties of the neurons and to determine their sensitivity to glutamate stimulation. Neurons were identified by sparse (1/5) green fluorescent protein (GFP) labelling, and qualitative morphological analysis by two-photon microscopy revealed a typical neuronal phenotype with long processes extending from the soma (Fig. 2d). To assess the intrinsic properties of these cells, we first tested for depolarization-induced $\mathrm{Ca}^{2+}$-entry by driving action potential firing. Neurons were whole-cell patch loaded with the membrane impermeant $\mathrm{Ca}^{2+}$-sensor Rhod-2 for $\mathrm{Ca}^{2+}$ imaging with 2photon microscopy (Fig. 2e). Stimulation trains (200 pA, $5 \mathrm{~ms} /$ pulse, $20 \mathrm{~Hz}, 5 \mathrm{~s}$ total) triggered action potentials (Fig. 2f-g) and temporally correlated with $\mathrm{Ca}^{2+}$ entry in the soma and dendrites as measured by an increase in Rhod-2 fluorescence (Fig. 2h), suggesting that the cells were both electrically excitable and expressed voltagegated $\mathrm{Ca}^{2+}$ channels. Lastly, we tested for functional expression of AMPA receptors in the membrane by exogenous glutamate stimulation. Glutamate was locally applied via a puff electrode $(200 \mu \mathrm{M}, 5 \mathrm{~s})$ and elicited reliable inward currents in cells voltage clamped at $V m=-60 \mathrm{mV}$. These currents were reversibly inhibited by bath application of the AMPA receptor antagonist CNQX $(10 \mu \mathrm{M})$, confirming that these currents were mediated by AMPA receptor opening (Fig. 2i-j). We further characterized astrocyte function by measuring apoE secretion after stimulation with the brain-penetrant Liver-X-Receptor (LXR) agonist GW3965 added to the circulating media before collecting tissue chamber and circulation media. After $96 \mathrm{~h}$, ELISA quantification confirmed a significant increase of secreted apoE in the chamber media of GW3965treated tissues while the concentration of apoE in the circulation media was below the detection limit of the
ELISA, and not different than control media (Fig. 2k), suggesting a lack of apoE transport across the endothelium in this model. These observations support astrocyte functionality via apoE secretion and a tight endothelium barrier as apoE does not cross the BBB in vivo [25]. To further assess astrocytes we immunostained cryopreserved longitudinal sections against aquaporin (AQ)4 and GFAP. AQ4 was localized in GFAP positive cells (yellow arrow) but also in GFAP negative cells (white arrow) similar to its localization patterns in human brain tissue (Sup. Fig. 2b). Finally, we assessed endothelial integrity by circulating 4 $k D a$ FITC-Dextran through the lumen of the bioengineered arterial NVU and measured a permeability of 8.3 $10 \mathrm{E}^{-9} \pm 6.06 \mathrm{E}^{-9} \mathrm{~cm} / \mathrm{s}$ (Sup. Fig 2c). Together, these data confirm that our bioengineered arterial NVU possesses both structural and functional characteristics of native neurons and astrocytes surrounding an arterial blood vessel with a tight endothelial barrier.

\section{Proof-of-principle for the utility of this bioengineered arterial NVU model to study neurodegenerative diseases}

In vitro models are used as research tools to address specific physiological or pathological questions that are not feasible to study in human subjects or are difficult to translate from animal models. Here we tested the hypothesis that our bioengineered arterial NVU could be used to study cerebrovascular contributions to $\mathrm{AD}$ or other neurodegenerative diseases. $\mathrm{AD}$ is the leading cause of dementia affecting over 50 million people worldwide with a global economic burden of over one trillion USD [26]. While extracellular plaques composed of beta-amyloid $(\mathrm{A} \beta)$ peptides, and intracellular neurofibrillary tangles are the classical neuropathological hallmarks of $\mathrm{AD}, 90 \%$ of $\mathrm{AD}$ patients also have some form of cerebral vessel disease, including vascular $A \beta$ deposition known as CAA [27]. We first used ELISA to quantify endogenous $A \beta$ secreted from iPSC neurons in the bioengineered arterial NVU in the tissue chamber and in the circulation media as well as in tissue lysates. We 
found $A \beta 40$ levels to be significantly higher than $A \beta 42$ levels in both the tissue chamber and circulating media (Fig. 3a). Specifically, $A \beta 42 / A \beta 40$ ratios were 0.078 and 0.0867 in the tissue chamber and circulating media, respectively, similar to the reported $A \beta 42 / A \beta 40$ ratio in human CSF [28] and blood [29]. Notably, A $\beta$ levels were higher in the tissue chamber than in the circulation media 4 days after the last media change (Fig. 3a), confirming that the tight endothelial barrier prevented diffusion of $A \beta$ between the two media compartments. Because $A \beta$ has been reported to be secreted by various cell types in vivo including EC [30], we confirmed that $\mathrm{A} \beta$ was predominantly secreted by neurons in our model by comparing tissues fabricated with and without neurons. ELISA quantification revealed that tissues with neurons had twice as much $A \beta 40$ in the tissue chamber than tissues composed only of EC and SMC (bipartite, $26.42 \mathrm{pg} / \mathrm{mL}$ ) and tissues composed of EC, SMC and astrocytes (tripartite, below detection limit of the ELISA, $<6 \mathrm{pg} / \mathrm{mL}$ ). Further, A $\beta 40$ concentration increased over time from 54.78 to $212.6 \mathrm{pg} / \mathrm{mL}$ after 1 week and 3 weeks in culture, respectively (Fig. 3b). A $\beta 42$ was only detectable in tissues with neurons, as $A \beta 42$ was below the detection limit of the ELISA $(10 \mathrm{pg} / \mathrm{mL})$ in bipartite and tripartite tissues lacking neurons. In tissue chamber media of bioengineered arterial NVU, A $\beta 42$ levels were $28.85 \mathrm{pg} /$ $\mathrm{mL}$ after 1 week (one sample below detection limit) and $15.56 \mathrm{pg} / \mathrm{mL}$ after 3 weeks in culture, respectively (Fig. 3c). In the circulation media, $A \beta 40$ and $A \beta 42$ were only detectable in tissues with neurons compared to tissues lacking neurons, where $A \beta 40$ and $A \beta 42$ levels were below the detection limit of the ELISAs (Sup. Fig 3a-b).

We next analyzed $A \beta$ deposition within the arterial NVU in RIPA, corresponding to interstitial and cellular fractions, and $\mathrm{GuHCl}$-soluble fractions, corresponding to the fraction containing insoluble $A \beta$ fibrils. We confirmed the presence of $A \beta 40$ in RIPA fraction in all 18 tissues tested, and $A \beta 42$ in 17 of the 18 tissues with most but not all RIPA lysates containing more $A \beta 40$ than A 342 (Fig. 3d). Similarly, A $\beta 40$ levels were significantly higher than A 442 in GuHCL-soluble lysates with 9 tissues (50\% of the total tissues analyzed) positive for A $\beta 40$ and only 2 tissues (11\%) above the ELISA detection limit for $A \beta 42$ (Fig. 3d). These results suggest that $A \beta 40$ accumulates to a greater extent than $A \beta 42$ in this model, which could be explained either by higher secretion of $A \beta 40$ as suggested in Fig. 3a, or by increased retention of $A \beta 40$ in the tissue. To test this hypothesis, we determined whether the level of $A \beta$ in the circulation media correlated with the level in the tissue chamber, reasoning that if increased $A \beta 40$ secretion causes the enhanced $A \beta 40$ accumulation, $A \beta 40$ and $A \beta 42$ levels in the tissue chamber and circulation media should correlate. However, if $A \beta 40$ is more readily retained in the tissue,
A $\beta 42$ levels in the tissue chamber and circulation media should correlate but $A \beta 40$ levels should not. While A $\beta 40$ levels in the circulation media were independent of those in the tissue chamber $\left(R^{2}=0.00218, p=0.8317\right)$, A $\beta 42$ levels in the circulating media and tissue chamber significantly correlated $\left(R^{2}=0.2208, p=0.0183\right.$ ), (Fig. 3ef), suggesting that $A \beta 40$ preferentially accumulates in the NVU. We further confirmed that the origin of accumulated vascular $\mathrm{A} \beta$ was neuronal by comparing tissues with and without neurons. While $A \beta 40$ and $A \beta 42$ tissue concentrations increased over time in tissues with neurons ( 1 vs 3 week), $A \beta 40$ and $A \beta 42$ levels in tissues lacking neurons were below the detection limit of the ELIS As (Fig. 3g-h). We further investigated $A \beta$ tissue localization by immunostaining cryopreserved longitudinal tissue sections against $A \beta$ fibrils (OC-fibril) or against $A \beta$ 1-16 (6E10) in comparison to human brain samples. Staining against OC-fibril showed extracellular deposition within the $\beta$-tub III (neuron marker) positive layers similar to the signal in human brain (white arrow), but also deeper within the tissue around the remaining of the scaffold (blue arrow) (Sup. Fig. 4a). Staining against 6E10 showed both extracellular signal (white arrow) and co-localization with the neuron marker MAP 2 (yellow arrow) like observed in human brain (Sup. Fig. 4b).

We also used immunoblotting to test if endogenous phosphorylated tau ( $\mathrm{p}$-tau), the first constituent of neurofibrillary tangles, was detectable in our bioengineered arterial NVU. We confirmed the presence of $\mathrm{p}$-tau in tissues with neurons using two different p-tau antibodies CP13 (pSer202) and PHF1 (pSer396/Ser404) as well as the early tangle marker AT8 (pSer202/Thr205) in comparison to a marker of total tau (DA9). As expected, tissues lacking neurons were negative for AT8, CP13 and PHF1, strongly supporting lack of p-tau (Fig. 3i). We also observed many cross-reactive bands using the total tau marker DA9 with distinct patterns in tissues with and without neurons. The p-tau/total tau ratio was calculated and graphed to show bioengineered arterial NVU variation (Fig. 3i). Finally, we immunostained longitudinally cut cryopreserved tissues against phospho-tau (AT8) and the neuronal marker MAP 2. Staining against AT8 showed both neuronal localization (yellow arrow) but also extracellular diffuse deposition (white arrow) similar to the signal observed in human brain (Sup. Fig. 3c). In addition, we observed a signal associated with the remaining scaffold (blue arrow) suggesting unspecific binding of AT8 to PGA or PCL. Taken together, these results support the potential utility of this model to study mechanisms related to vascular $A \beta$ and tau deposition.

\section{Characterization of NF-L, GFAP, total tau and UCH-L1 biomarkers in the engineered NVU}

The possibility to study fluid biomarkers using a humanbased in vitro model of the cerebrovasculature has, to 


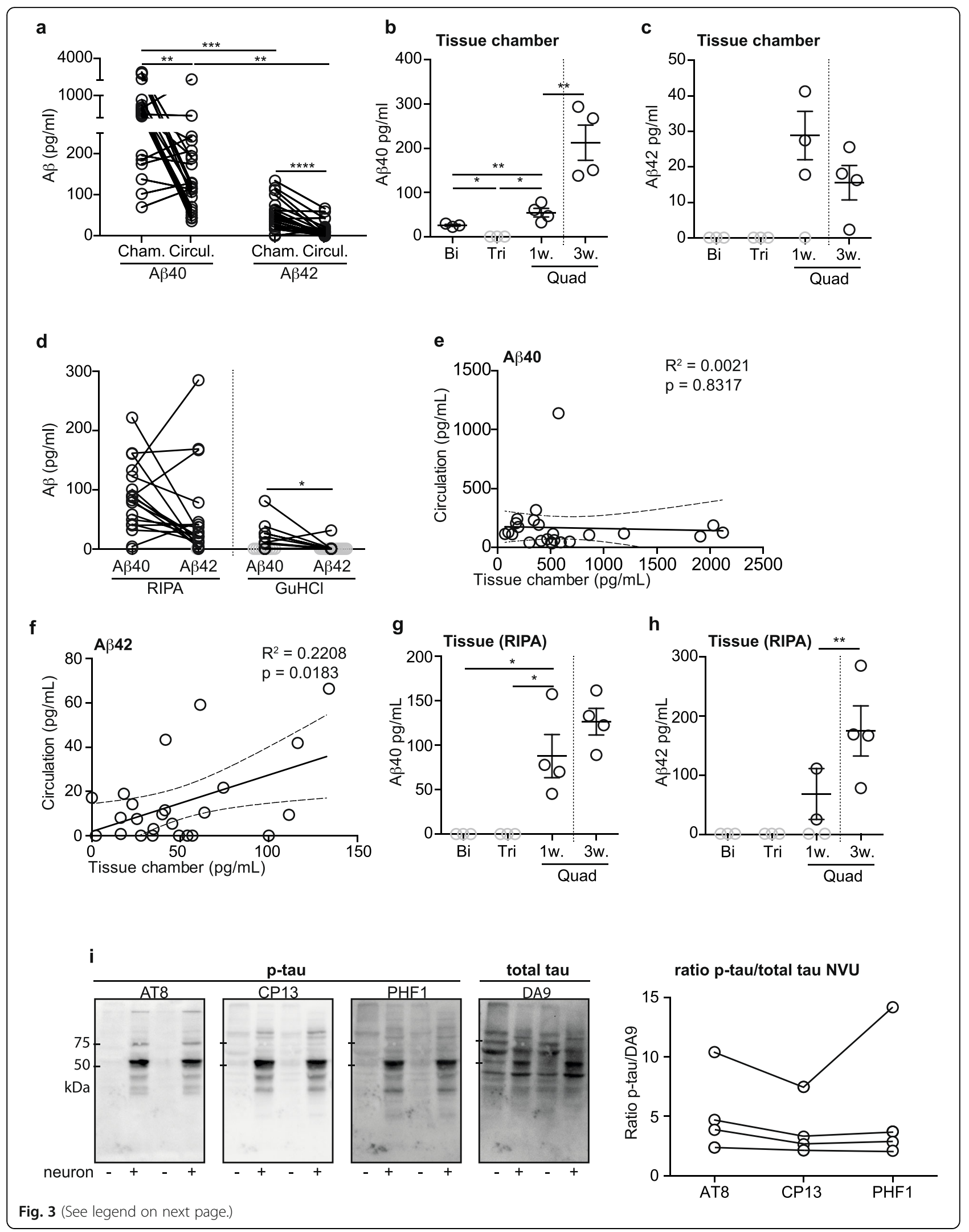




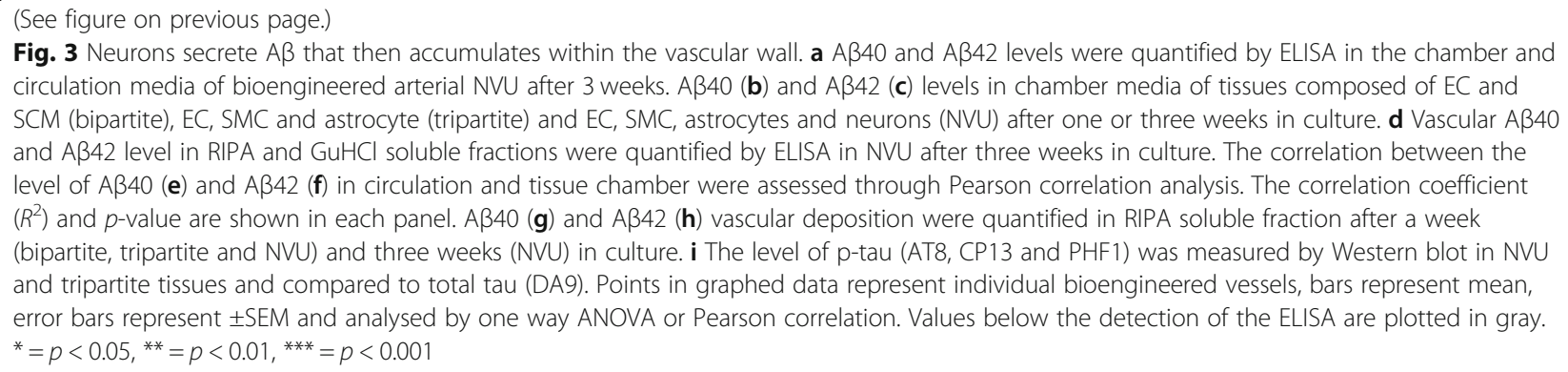

Fig. 3 Neurons secrete $A \beta$ that then accumulates within the vascular wall. a $A \beta 40$ and $A \beta 42$ levels were quantified by ELISA in the chamber and circulation media of bioengineered arterial NVU after 3 weeks. A 340 (b) and A 342 (c) levels in chamber media of tissues composed of EC and SCM (bipartite), EC, SMC and astrocyte (tripartite) and EC, SMC, astrocytes and neurons (NVU) after one or three weeks in culture. $\mathbf{d}$ Vascular A 40 and A $A 42$ level in RIPA and GuHCl soluble fractions were quantified by ELISA in NVU after three weeks in culture. The correlation between the level of $A \beta 40(\mathbf{e})$ and $A \beta 42(\mathbf{f})$ in circulation and tissue chamber were assessed through Pearson correlation analysis. The correlation coefficient $\left(R^{2}\right)$ and $p$-value are shown in each panel. $A \beta 40(\mathbf{g})$ and $A \beta 42(\mathbf{h})$ vascular deposition were quantified in RIPA soluble fraction after a week (bipartite, tripartite and NVU) and three weeks (NVU) in culture. i The level of p-tau (AT8, CP13 and PHF1) was measured by Western blot in NVU and tripartite tissues and compared to total tau (DA9). Points in graphed data represent individual bioengineered vessels, bars represent mean, error bars represent \pm SEM and analysed by one way ANOVA or Pearson correlation. Values below the detection of the ELISA are plotted in gray. ${ }^{*}=p<0.05,{ }^{* *}=p<0.01,{ }^{* * *}=p<0.001$

our knowledge, not yet been investigated. Nevertheless, a biofidelic human model would have undeniable translational advantages over animal models to validate or discover novel biomarkers of disease. As a proof-ofprinciple, we tested whether total tau, neurofilament light (NF-L), ubiquitin carboxy-terminal hydrolase L1 (UCH-L1) and glial fibrillary acidic protein (GFAP) could be quantified in both chamber and circulation media using an ultrasensitive single molecule immunoassay (Simoa). After 3 weeks in culture and 4 days after the last media change in the circulation loop, we observed that the levels of all four biomarkers were significantly higher in the tissue chamber compared to the circulation media (Fig. 4a-d). These results were confirmed by calculating the circulation:chamber ratio (Fig. 4e), providing additional support for a tight endothelial barrier in our model. Interestingly, we confirmed a positive correlation between chamber and circulation media for both NF$\mathrm{L}$ and GFAP but no correlation for total tau and UCH-L1 (Fig. 4f-i). Finally, we used correlation analyses to compare the level of each biomarker in the tissue chamber and circulation media. For this, we selected two pairs of biomarkers for dependence analysis, namely NF-L:total tau and GFAP:A $\beta 40$ for which we provide the plotted data (Fig. 5a and c) and a summary table for all other analyses (Fig. 5b and d). Dependence analyses show that levels of total tau, NF-L, GFAP and UCH-L1 strongly and positively correlate with each other in the tissue chamber. A 340 only correlated with GFAP and A $\beta 42$ only correlated weakly and negatively with total tau (Fig. 5a-b). In the circulation media, total tau levels correlated positively with NF-L, GFAP and UCH-L1 levels, and NF-L levels correlated with GFAP levels. A $\beta 40$ levels correlated positively with total tau, NF-L and GFAP levels but not with UCH-L1 or A $\beta 42$ levels (Fig. $5 \mathrm{c}-\mathrm{d}$ ). Taken together, these results support the potential utility of this model to study cerebral biomarkers and their dependence or independence to each other for mechanistic studies.

\section{Discussion}

The interaction between neurons and the cerebrovasculature is essential for brain function and health. This neuronal-vascular interplay regulates cerebral blood flow and blood-brain exchange, and dysfunction of the NVU is associated with several neurological diseases [31]. As the lack of robust and physiologically relevant models of the human NVU is recognized to be a major roadblock for understanding the cerebrovasculature in health and disease, interest in modeling the human cerebrovasculature, particularly for capillary models, is surging. Here, we combined primary human vascular cells and human iPSC-derived neurons using a tissue engineering approach to produce a functional, human, large vessel, perfusible NVU model that can be cultured under nativelike flow conditions for at least 21 days.

Early NVU models focused on modeling either vascular or neuronal components in isolation and under static conditions. Brain-derived EC, cultured alone or cocultured with other cerebrovascular cells, often in transwells, are typically used to evaluate the ability of therapeutic agents to cross the BBB [32]. Animal- or humanderived neurons cultured in regular culture dishes lack the $3 \mathrm{D}$ cellular organization that regulates neuronal function and many key cellular processes in vivo. Importantly, it has become clear in the last decade that cells sense and respond to the dimensionality and rigidity of their environment, and these qualities cannot be modeled using classical tissue culture methods [33]. Newer approaches often use multicellular spheroid systems consisting of human primary or iPSC-derived EC, pericytes, astrocytes and neurons that are cultured into multicellular BBB- and/or brain-organoid structures [34-38]. These organoids can be maintained for extensive time in culture, holding great promise to study neuronal functions. However, controlled perfusion through a validated vascular lumen to study blood-tobrain and brain-to-blood transport has not yet been possible, and neurons within the center of such organoids may be nutrient starved. To address this gap, several groups have developed capillary-like NVU models using microfluidic systems. These platforms offer control over luminal flow, but focus on BBB function over neuronal function in the NVU, as the vascular cells are separated from neurons either by a porous membrane or cultured in different chips that are linked together [13, 15, 39]. Here, we opted to use bioengineering techniques to co- 


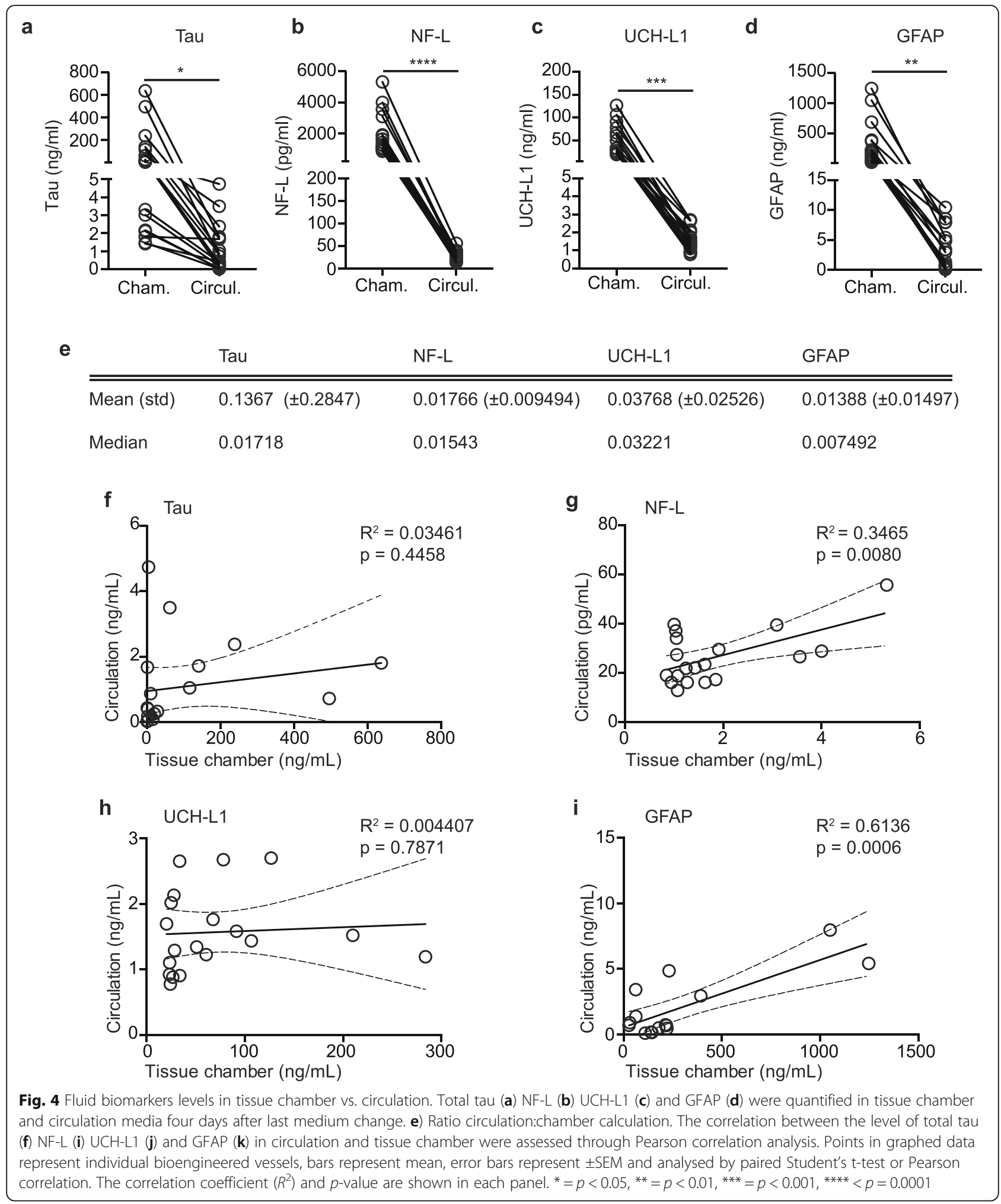

culture human EC, SMC, astrocytes and neurons to model the NVU structure of cortical penetrating arteries. Our approach offers the possibility to control luminal perfusion, assess bidirectional (blood-to-brain and brain- to-blood) trans-endothelial transport, as well as assess neuronal, glial and endothelial functions in a model where cell types are grown in close proximity to each other within an endogenously secreted extracellular 


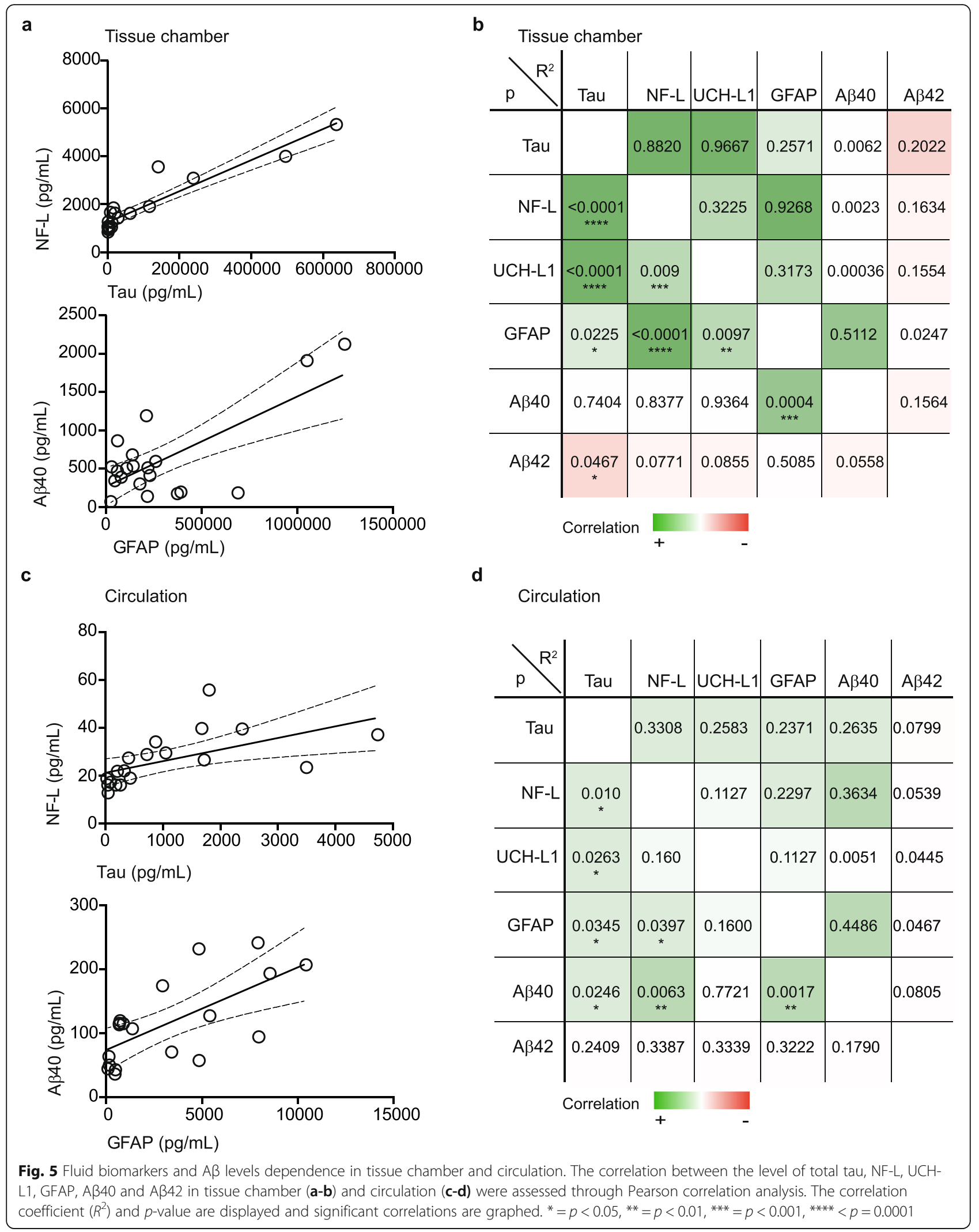


matrix. After 21 days in culture with luminal flow, we demonstrated that abluminal neurons have histological, biochemical and electrical functions. While the measured electrical properties - in particular the resting potential - suggest that neurons in our model were not fully mature at the time of analysis, they are comparable in their electrophysiology to that previously measured in brain organoids [14]. The endothelial barrier represents an important feature of the NVU, and most previous studies use either FITC-dextran or trans endothelial electrical resistance (TEER) measurement to assess BBB integrity $[40,41]$. Here we confirmed the integrity of the endothelium barrier using $4 \mathrm{kDa}$ FITC-dextran. We calculated a permeability of $8.310 \mathrm{E}^{-9} \pm 6.06 \mathrm{E}^{-9} \mathrm{~cm} / \mathrm{s}$, similar to values we previously reported in bioengineered tissues composed of EC and SMC cultured in the presence $\left(4.910 \mathrm{E}^{-7} \mathrm{~cm} / \mathrm{s}\right)$ or absence $\left(7.010 \mathrm{E}^{-7} \mathrm{~cm} / \mathrm{s}\right)$ of astrocytes [12] or to published values (reviewed in [42]). However, dextran and TEER assays require external non-physiological chemicals to be circulated through the model, or electrical probes to be placed in the vascular lumen that could potentially lead to cellular disturbance. Thus, we also confirmed in independent tissues that astrocyte-secreted apoE remains in the tissue chamber and does not cross the endothelial barrier as documented in vivo [25] and that other fluid biomarkers show differential levels in the tissue chamber compared to the circulation media. Specifically, the differential concentrations of $A \beta 40, A \beta 42$, tau, GFAP, NF-L and $\mathrm{UCH}-\mathrm{L} 1$ between the two compartments confirm tight endothelial barrier formation with specific transport mechanisms to be analyzed in future studies.

Although NVU models are being developed at a rapid pace for potential use in the study of neurodegenerative diseases, only a few studies have provided evidence supporting successful disease modeling $[43,44]$. Our laboratory previously generated a vascular model composed of $\mathrm{EC}, \mathrm{SMC}$ and astrocytes that can be used to study CAA, a component of $\mathrm{AD}$, but the absence of neurons in our previous studies required injection of exogenous recombinant $\mathrm{A} \beta$ into the tissue chamber to initiate CAA [12]. Recently, Shin and colleagues developed a microfluidic AD model composed of neurons, astrocytes and EC to study A $\beta$-induced BBB damage [44]. Although representing an undeniable step forward in developing an in vitro model of vascular $\mathrm{AD}$, this model requires the overexpression of APP/PSEN1 genes with early onset familial AD (FAD) mutations due to short culture times, which may limit the physiologic relevance of the model to study late onset $\mathrm{AD}$ that accounts for the majority of total AD cases [45]. Here, we used neurons without FAD mutations and maintained engineered tissues in culture for up to 21 days to ensure $A \beta$ secretion and deposition that closely mimics observations in the human cerebrovasculature in vivo. A human-based model of CAA is highly relevant, as $A \beta$ deposition within the vascular wall is present in up to $40 \%$ of non-cognitively declined elderly brains and in $80 \%$ of late onset $\mathrm{AD}$ brains $[46,47]$. Using this approach, we have shown that $A \beta 40$ fibrils preferentially accumulate to a greater extent in the vasculature compared to $A \beta 42$, in parallel to what has been shown in vivo $[48,49]$. These results are concomitant to our earlier findings where we showed that recombinant $\mathrm{A} \beta 42$ is more amenable to lipoprotein (apoE and high-density lipoprotein)-mediated transit across and removal from the vascular wall of bioengineered cerebral vessels composed of EC and SMC with or without astrocytes than recombinant $A \beta 40$ [12]. In our model, according to dependence calculations, the enhanced deposition of $A \beta 40$ might not only result from an enhanced $A \beta 40$ production in our model but also be due to an altered vascular transport or diffusion compared to $A \beta 42$. Interestingly, $A \beta 40$ levels are only elevated in GuHCL but not in RIPA fractions, suggesting that $A \beta 40$ may preferentially bind to the extracellular matrix rather than accumulating intracellularly or in the interstitial fluid. Future studies are needed to fully understand the mechanisms that govern differential transport and deposition of $A \beta 40$ and $A \beta 42$ within the vascular wall. For example, we did not test whether tissue or media $A \beta$ levels depend on $A \beta$ transporter (i.e, LRP1, RAGE, glycoprotein) or $A \beta$ binding protein (i.e. clusterin, apoE) levels. Notably, we also demonstrated that endogenous tau protein was phosphorylated in our model. Together, these results establish a promising framework to use this NVU model as an AD-relevant research tool to investigate the mechanisms of human NVU function in healthy and diseased states.

Research on fluid biomarkers for several neurological diseases is advancing at a tremendous pace, with intense interest in developing blood tests [50]. Here we demonstrated that several neuronal and glial biomarkers used in clinical studies could be detected in both the tissue chamber and circulation media. Although the absolute biomarker levels were different compared to in vivo conditions, the ratio of circulation:chamber levels was comparable to human blood: CSF ratios [51-55]. Thus, our model offers the potential to study how specific protein levels correlate in "brain" to "blood" compartments to understand the dependence and independence among biomarkers, recognizing that our model lacks peripheral catabolic pathways that govern clearance and excretion of blood proteins from the body. Using this approach, we found that in the tissue chamber, tau and NF-L levels do not correlate with $A \beta 40$ levels, but the levels of these biomarkers do correlate in the circulation media. This suggests that blood tau and NF-L levels are independent of the amount of $A \beta 40$ produced, but appear to depend 
on the amount of $\mathrm{A} \beta 40$ retained in the vascular wall. $\mathrm{Fu}$ ture studies are required to fully define these relationships and compare them to in vivo data. Of particular interest would be measures of NF-L and tau levels in CAA patients. Importantly, the correlation analyses presented here only represents the statistical dependence between two variables and further studies are required to fully define the relationships and compare them to in vivo data.

This study has several limitations. The stiffness of the current scaffold material used in this model prevents studying vascular compliance, which is an important outcome measure of neurovascular coupling. Refinement of a more suitable scaffold material may enable the study of SMC contraction and relaxation in response to neuronal activity or circulating stimuli, which is an important objective given that several neurodegenerative disease patients have altered CBF $[8,56,57]$ and CBF regulation happens both in arterioles and in capillaries [7]. Another limitation is that this study used cells derived from umbilical cord, due to the slow growth rate and expense of primary human cerebrovascular cells. Although cord cells clearly do not reflect the physiology of brain vasculature, we and others have previously demonstrated that HUVECs express BBB marker proteins when co-cultured with astrocytes and thus may be suitable as a cost-effective cellular source for model development [12, 58, 59]. That apoE, which does not cross the BBB [25], is restricted to the abluminal chamber in our model supports the conclusion that HUVEC can form a functional endothelial barrier. The choice to use primary cells over iPSC-derived cells was motivated by the accessibility of primary human EC, SMC and astrocytes, and because we previously reported the feasibility to bioengineer cerebral vessels using these cells [12, 19]. Future studies will be required to develop tissues made entirely from patient-derived iPSCs for personalized medicine applications. Importantly, as iPSCs will be specific to each individual donor, they have the potential to be highly variable between donor batches, and studies using isogenic iPSC sets are encouraged. Another limitation is that the protocol used here for iPSC neuronal differentiation is known to generate a mixed population of cells positive for the neuronal markers MAP 2 and $\beta$-tubulin-III (>75\%) and cells positive for GFAP $(<25 \%)$, as previously reported [20]. Despite these limitations, our study is a proof-of-concept demonstration that a cerebral arterial NVU can be engineered in vitro as a potentially relevant tool to study neurodegenerative diseases.

\section{Conclusion}

Our arterial NVU model represents an important step toward the development of a human-based translational in vitro model of large cerebral vessels. It could be a useful research tool for the study of cerebrovascular function in both physiological and pathophysiological conditions. In particular, as our model offers the possibility to study transport from the brain to the circulation and vice versa, it also has the potential to be a novel and relevant platform for drug development targeting both neuronal and vascular function, and opens up the possibility to study neuronal and glial biomarkers.

\section{Supplementary Information}

The online version contains supplementary material available at https://doi. org/10.1186/s13024-020-00418-z

Additional file 1: Supplemental Figure 1. Histological structure of human arterial NVU. a) Human cortex (Brodmann area 9) were stained against a) VWF (EC marker), aSMA (SMC marker) and GFAP (astrocyte marker) and against b) MAP 2 (neuron marker), aSMA (SMC marker) and GFAP (astrocyte marker) to visualize the cerebrovasculature.

Additional file 2: Supplemental Figure 2. Histological characterization and function of bioengineered arterial NVU. a) The histological structure of the arterial NVU was assessed by H\&E staining. b) The astrocytes in arterial NVU were further analyzed with immunostaining against aquaporin 4 (AQ4). Barrier integrity was assessed by measuring permeability of $250 \mu \mathrm{g} / \mathrm{ml}$ of $4 \mathrm{kDa}$ FITC-dextran circulated through the lumen for $2 \mathrm{~h}$. Points in graphed data represent individual bioengineered vessels, bars represent mean, error bars represent \pm SEM. $A B L=$ antelumen.

Additional file 3: Supplemental Figure 3. $A \beta 40$ and $A \beta 42$ concentration in circulation media. (a) A 340 and (b) $A \beta 42$ levels in circulation media of tissue composed of EC and SCM (bipartite), EC, SMC and astrocyte (tripartite) and EC, SMC, astrocytes and neurons (NVU) after 1 or 3 weeks in culture were quantified by ELISA. Points in graphed data represent individual bioengineered vessels, bars represent mean, error bars represent \pm SEM and analysed by one way ANOVA. Values below the detection of the ELISA are plotted in gray. ${ }^{*}=p<0.05,{ }^{* *}=p<0.01,{ }^{* *}=$ $p<0.001$.

Additional file 4: Supplemental Figure 4. $A \beta$ and $p$-tau histology. Cryopreserved bioengineered arterial NVU were cut longitudinally to show a cross-section of the NVU wall. a) Immunohistochemistry against $O C$ fibril confirmed the deposition of $A \beta$ fibril both in the neuron layer ( $\beta$-tub III positive, white arrow) as well as deeper in the vascular wall (blue arrows). b) The expression of $A \beta$ was further investigated by staining against $A \beta$ 1-16. Positive signal was found both deep in the vascular wall (white arrow) as well as co-localized within MAP 2 positive cells (yellow arrow). c) Immunohistochemistry against AT8 suggest the deposition of p-tau both within neuron (MAP 2 positive, yellow arrow) as well as extracellular (white arrow), purple arrow shows the remaining of the scaffold. Human brains were used as representative staining pattern. $\mathrm{ABL}=$ albumen.

\section{Acknowledgements}

This work was supported by Weston Brain Institute Rapid Response grants (RR182093 \& RR150048), and a BrightFocus Foundation Alzheimer's Disease Award awarded to JR. A Cure Alzheimer Fund, NSERC discovery, and Canadian Consortium of Neurodegeneration and Aging (CCNA) grants awarded to CLW. JR was further supported by Michael Smith Foundation for Health Research (MSFHR)/CCNA and UBC Faculty of Medicine Bluma Tischler fellowships. EBB was supported by a UBC Four Year Doctoral Fellowship and a Canadian Institutes of Health Research Doctoral Fellowships. EMR was supported by an Alzheimer Society of Canada Doctoral Award and a University of British Columbia Faculty of Medicine Graduate Award. NLW was supported by postdoctoral fellowships from MSFHR and the Canadian Institutes for Health Research (CIHR, Banting Award). BAM holds grants from CIHR (\#FDN148397) and Fondation Leducq (15CVD02). PS was supported by a postdoctoral fellowship from the German Research Foundation (SE 2608/1- 
1). SC and L-PZ were supported through a Canadian Excellence Research Chair to MJF.

\section{Authors' contributions}

$J R$ and CLW designed the research with input from MF, NLW and SC. JR, NLW, LPZ, SC, SS, EMM, PS, MG, EMR, TMC and JF performed the experiments and analyzed the data. JR, CLW, MF and BMV obtained the funding. JR, CLW, EMR and TMC drafted the manuscript and all authors reviewed and approved it

\section{Availability of data and materials}

Raw data can be obtained from corresponding authors.

\section{Ethics approval and consent to participate}

All experiments were conducted under an approved clinical protocol from the UBC Clinical Ethics Research Board after obtaining written informed consent from all subjects (H13-02719: isolation of vascular cells). Human brain tissues were received from the Harvard Brain Tissue Resource Center under UBC Clinical Research Ethics Board protocol C04-0595.

\section{Consent for publication}

Not applicable.

\section{Competing interests}

The authors declare no competing interests.

\section{Author details}

'Department of Pathology and Laboratory Medicine, University of British Columbia, Vancouver, British Columbia V6T 1Z3, Canada. ${ }^{2}$ Djavad Mowafaghian Centre for Brain Health, University of British Columbia, 2215 Wesbrook Mall, Vancouver, British Columbia V6T 1Z3, Canada. Institute of Clinical Chemistry, University hospital Zurich, 8000 Zurich, Wagistrasse 14, CH-8952 Schlieren, Switzerland. ${ }^{4}$ Centre for Applied Neurogenetics, University of British Columbia, Vancouver, British Columbia V6T 1Z3, Canada. Institute of Neurogenetics, University of Luebeck, 23562 Luebeck, Germany.

${ }^{6}$ Laboratory for Neurogenetics \& Neuroscience, McKnight and Fixel Institutes, University of Florida, Gainesville 32610, USA. ${ }^{7}$ School of Biomedical Engineering, University of British Columbia, Vancouver, British Columbia V6T 1Z3, Canada. International Collaboration on Repair Discoveries, University of British Columbia, Vancouver, British Columbia V5Z 1M9, Canada.

Received: 8 June 2020 Accepted: 3 November 2020 Published online: 19 November 2020

\section{References}

1. Rolfe DF, Brown GC. Cellular energy utilization and molecular origin of standard metabolic rate in mammals. Physiol Rev. 1997;77:731-58.

2. Attwell D, Laughlin SB. An energy budget for signaling in the grey matter of the brain. J Cereb Blood Flow Metab. 2001;21:1133-45.

3. Kisler K, Nelson A, Montagne A, et al. Cerebral blood flow regulation and neurovascular dysfunction in Alzheimer disease. Nat Rev Neurosci. 2017;18: 419-34. https://doi.org/10.1038/nrn.2017.48.

4. ladecola C. Neurovascular regulation in the normal brain and in Alzheimer's disease. Nat Rev Neurosci. 2004:5:347-60.

5. Buxton RB, Frank LR. A model for the coupling between cerebral blood flow and oxygen metabolism during neural stimulation. J Cereb Blood Flow Metab. 1997;17:64-72.

6. Attwell D, et al. Glial and neuronal control of brain blood flow. Nature. 2010; 468:232-43.

7. Mishra A, et al. Astrocytes mediate neurovascular signaling to capillary pericytes but not to arterioles. Nat Neurosci. 2016;19:1619-27.

8. Hall CN, et al. Capillary pericytes regulate cerebral blood flow in health and disease. Nature. 2014;508:55-60.

9. Biesecker $\mathrm{KR}$, et al. Glial cell calcium signaling mediates capillary regulation of blood flow in the retina. J Neurosci. 2016;36:9435-45.

10. Sweeney MD, Sagare AP, Zlokovic BV. Blood-brain barrier breakdown in Alzheimer disease and other neurodegenerative disorders. Nat Rev Neurol. 2018:14:133-50

11. Sweeney MD, Kisler K, Montagne A, Toga AW, Zlokovic BV. The role of brain vasculature in neurodegenerative disorders. Nat Neurosci. 2018;21:1318-31. https://doi.org/10.1038/s41593-018-0234-x.
12. Robert J, et al. Clearance of beta-amyloid is facilitated by apolipoprotein $\mathrm{E}$ and circulating high- density lipoproteins in bioengineered human vessels. Elife. 2017;6:e29595. https://doi.org/10.7554/eLife.29595.

13. Maoz BM, et al. A linked organ-on-chip model of the human neurovascular unit reveals the metabolic coupling of endothelial and neuronal cells. Nat Biotechnol. 2018. https://doi.org/10.1038/nbt.4226.

14. Cakir B, et al. Engineering of human brain organoids with a functional vascular-like system. Nat Methods. 2019. https://doi.org/10.1038/s41592-0190586-5.

15. Vatine GD, et al. Human iPSC-Derived Blood-Brain Barrier Chips Enable Disease Modeling and Personalized Medicine Applications. Cell Stem Cell. 2019;24:995-1005.e6.

16. Winship IR. Cerebral collaterals and collateral therapeutics for acute ischemic stroke. Microcirculation. 2015;22:228-36.

17. Weber SA, Patel RK, Lutsep HL. Cerebral amyloid angiopathy: diagnosis and potential therapies. Expert Rev Neurother. 2018;18:503-13.

18. Button EB, Gilmour M, Cheema HK, Martin EM, Agbay A, Robert J, Wellington CL. Vasoprotective functions of high-density lipoproteins relevant to Alzheimer's disease are partially conserved in Apolipoprotein Bdepleted plasma. Int J Mol Sci. 2019;20:462.

19. Robert J, et al. High-density lipoproteins suppress AB-induced PBMC adhesion to human endothelial cells in bioengineered vessels and in monoculture. Mol Neurodegener. 2017;12:60.

20. Shi Y, Kirwan P, Livesey FJ. Directed differentiation of human pluripotent stem cells to cerebral cortex neurons and neural networks. Nat Protoc. 2012; 7:1836-46.

21. Seibler $P$, et al. Iron overload is accompanied by mitochondrial and lysosomal dysfunction in WDR45 mutant cells. Brain. 2018;141:3052-64.

22. Zhong $L$, et al. A rapid and cost-effective method for genotyping apolipoprotein E gene polymorphism. Mol Neurodegener. 2016;11:2.

23. Robert J, et al. A three-dimensional engineered artery model for in vitro atherosclerosis research. PLoS One. 2013:8:e79821.

24. Fan J, et al. Identification of a Chrysanthemic Ester as an Apolipoprotein E inducer in astrocytes. PLoS One. 2016;11:e0162384.

25. Linton MF, et al. Phenotypes of apolipoprotein B and apolipoprotein E after liver transplantation. J Clin Invest. 1991:88:270-81.

26. Christina P. World Alzheimer's report 2018. Alzheimers Dis Internations. 2018:2018:1-48. https://doi.org/10.1111/j.0033-0124.1950.24_14.x.

27. Snyder HM, et al. Vascular contributions to cognitive impairment and dementia including Alzheimer's disease. Alzheimers Dement. 2015:11:710-7.

28. Janelidze $S$, et al. CSF $A \beta 42 / A \beta 40$ and $A \beta 42 / A \beta 38$ ratios: better diagnostic markers of Alzheimer disease. Ann Clin Transl Neurol. 2016;3:154-65.

29. van Oijen M, Hofman A, Soares HD, Koudstaal PJ, Breteler MM. Plasma Aß140 and $A \beta 1-42$ and the risk of dementia: a prospective case-cohort study. Lancet Neurol. 2006;5:655-60.

30. Kitazume $S$, et al. Brain endothelial cells produce amyloid $\beta$ from amyloid precursor protein 770 and preferentially secrete the O-glycosylated form. J Biol Chem. 2010;285:40097-103.

31. Sagare AP, Bell RD, Zlokovic BV. Neurovascular dysfunction and faulty amyloid $\beta$-peptide clearance in Alzheimer disease. Cold Spring Harbor Perspect Med. 2012;2(10):a011452. https://doi.org/10.1101/cshperspect. a011452.

32. Helms $\mathrm{HC}$, et al. In vitro models of the blood-brain barrier: an overview of commonly used brain endothelial cell culture models and guidelines for their use. J Cereb Blood Flow Metab. 2016;36:862-90.

33. Potjewyd G, Moxon S, Wang T, Domingos M, Hooper NM. Tissue engineering 3D neurovascular units: a biomaterials and bioprinting perspective. Trends Biotechnol. 2018;36:457-72.

34. Urich $E$, et al. Multicellular self-assembled spheroidal model of the blood brain barrier. Sci Rep. 2013;3:1500.

35. Cho CF, et al. Blood-brain-barrier spheroids as an in vitro screening platform for brain-penetrating agents. Nat Commun. 2017:8:1-14.

36. Pasca SP. Assembling human brain organoids. Science. 2019;363:(6423):1267. https://doi.org/10.1126/science.aau5729.

37. Pham MT, et al. Generation of human vascularized brain organoids. Physiol Behav. 2017:176:139-48.

38. Song $L$, et al. Assembly of human stem cell-derived cortical spheroids and vascular spheroids to model 3-D brain-like tissues. Sci Rep. 2019;9:5977.

39. Adriani G, Ma D, Pavesi A, Kamm RD, Goh ELK. A 3D neurovascular microfluidic model consisting of neurons, astrocytes and cerebral endothelial cells as a blood-brain barrier. Lab Chip. 2017;17:448-59. 
40. Jeong $\mathrm{S}$, et al. A three-dimensional arrayed microfluidic blood-brain barrier model with integrated electrical sensor array. IEEE Trans Biomed Eng. 2018; 65:431-9.

41. Wang YI, Abaci HE, Shuler ML. Microfluidic blood-brain barrier model provides in vivo-like barrier properties for drug permeability screening Biotechnol Bioeng. 2017;114:184-94.

42. Bhalerao $A$, et al. In vitro modeling of the neurovascular unit: advances in the field. Fluids Barriers CNS. 2020;17:22.

43. Choi SH, Kim YH, Quinti L, Tanzi RE, Kim DY. 3D culture models of Alzheimer's disease: a road map to a "cure-in-a-dish". Mol Neurodegener. 2016;11:75.

44. Shin Y, Choi SH, Kim E, Bylykbashi E, Kim JA, Chung S, Kim DY, Kamm RD, Tanzi RE. Blood - brain barrier dysfunction in a 3D in vitro model of Alzheimer's disease. Adv Sci. 2019;6:1900962. https://doi.org/10.1002/advs. 201900.

45. Lane CA, Hardy J, Schott JM. Alzheimer's disease. Eur J Neurol. 2018;25:5970.

46. Attems J, Jellinger KA. The overlap between vascular disease and Alzheimer's disease--lessons from pathology. BMC Med. 2014;12:206

47. Biffi A, Greenberg SM, et al. J Clin Neurol (Seoul, Korea). 2011;7:1-9.

48. Gravina $S A$, et al. Amyloid $\beta$ protein $(A \beta)$ in Alzheimeri's disease brain. J Biol Chem. 1995:270:7013-6.

49. Iwatsubo $\mathrm{T}$, et al. Visualization of a beta $42(43)$ and a beta 40 in senile plaques with end-specific a beta monoclonals: evidence that an initially deposited species is a beta 42(43). Neuron. 1994;13:45-53.

50. Simrén J, Ashton NJ, Blennow K, Zetterberg H. An update on fluid biomarkers for neurodegenerative diseases: recent success and challenges ahead. Curr Opin Neurobiol. 2019;61:29-39.

51. Mondello S, et al. Ubiquitin Carboxy-terminal hydrolase L1 (UCH-L1) is increased in cerebrospinal fluid and plasma of patients after epileptic seizure. BMC Neurol. 2012;12:1.

52. Jany PL, et al. CSF and blood levels of GFAP in Alexander disease. eNeuro. 2015;2(5): ENEURO.0080-15.2015. https://doi.org/10.1523/ENEURO.0080-15. 2015.

53. Mielke MM, et al. Plasma and CSF neurofilament light: relation to longitudinal neuroimaging and cognitive measures. Neurology. 2019;93: E252-60.

54. Staffaroni AM, et al. Association of Blood and Cerebrospinal Fluid tau Level and other biomarkers with survival time in sporadic Creutzfeldt-Jakob disease. JAMA Neurol. 2019;76:969-77.

55. Zetterberg $\mathrm{H}$, et al. Plasma tau levels in Alzheimer's disease Plasma tau levels in Alzheimer's disease. Alzheimers Res Ther. 2013:5-9. https://doi.org/ 10.1186/alzrt163.

56. de la Torre JC. Cerebral hemodynamics and vascular risk factors: setting the stage for Alzheimer's disease. J Alzheimers Dis. 2012;32:553-67.

57. Nelson AR, Sagare MA, Yaoming W, Kassandra K, Zhen Z, Zlokovic Berislav V. Channelrhodopsin excitation contracts brain Pericytes and reduces blood flow in the aging mouse brain in vivo. Front Aging Neurosci. 2020;12:108. https://doi.org/10.3389/fnagi.2020.00108.

58. Wu C-C, et al. Human umbilical vein endothelial cells protect against hypoxic-ischemic damage in neonatal brain via stromal cell-derived factor 1/C-X-C chemokine receptor type 4. Stroke. 2013;44:1402-9.

59. Akiyama $\mathrm{H}$, et al. Blood-brain barrier formation of grafted human umbilical vein endothelial cells in athymic mouse brain. Brain Res. 2000;858:172-6.

\section{Publisher's Note}

Springer Nature remains neutral with regard to jurisdictional claims in published maps and institutional affiliations.

Ready to submit your research? Choose BMC and benefit from:

- fast, convenient online submission

- thorough peer review by experienced researchers in your field

- rapid publication on acceptance

- support for research data, including large and complex data types

- gold Open Access which fosters wider collaboration and increased citations

- maximum visibility for your research: over $100 \mathrm{M}$ website views per year

At BMC, research is always in progress.

Learn more biomedcentral.com/submissions 